\title{
Unsteady flow in trailing vortices
}

\author{
By S. I. GREEN ${ }^{1}$ AND A. J. ACOSTA A $^{2}$ \\ ${ }^{1}$ University of British Columbia Vancouver BC V6T 1W5, Canada. \\ ${ }^{2}$ California Institute of Technology Pasadena, CA 91125, USA
}

(Received 2 October 1989 and in revised form 16 November 1990)

The instantaneous velocity distribution in trailing vortices generated by lifting hydrofoils has been measured in the Low Turbulence Water Tunnel at the California Institute of Technology. Two different rectangular planform hydrofoils with small aspect ratios were tested. Double-pulsed holography of injected microbubbles, which act much as Lagrangian flow tracers, was used to determine instantaneous axial and tangential velocities. Measurements were made at various free-stream velocities, angles of attack, and downstream distances. The vortex core mean axial velocity is consistently greater than the free-stream velocity near the hydrofoil trailing edge, and decreases with downstream distance. The mean axial velocity is strongly Reynolds-number dependent.

Axial flow in the trailing vortex is highly unsteady for all the flow conditions studied; peak-to-peak fluctuations on the centreline as large as the free-stream velocity have been observed. The amplitude of these fluctuations falls rapidly with increasing distance from the centreline. For an angle of attack of $10^{\circ}$ the fluctuations consist of both 'fast' and 'slow' components, whereas for $\alpha=5^{\circ}$ only 'fast' fluctuations have been observed. Peak decelerations of the centreline fluid occur with amplitude comparable to the maximum centripetal acceleration around the centreline. Certain unusual structures of the vortex core - regions in which the flow direction quickly diverges from the free-stream direction, and then equally quickly recovers - have been labelled 'vortex kinks.'

\section{Introduction}

Tip vortex flow is an important aspect of many engineering problems. For example, all tip vortices represent lifting inefficiencies because they are caused by pressure equalization near the tip of a lifting surface. Furthermore, small aircraft which accidentally fly into the tip vortices generated by larger planes experience a destabilizing rolling or pitching moment; Chigier (1974) has reported that over 100 serious injuries and deaths have occurred as a result of such encounters. Helicopter rotor tip vortices are major noise sources, and propeller tip vortex cavitation causes both undesirable noise and wear.

Two features of trailing vortices make them inimical to most measurement techniques. Trailing vortices in a water or wind tunnel 'meander' or 'wander' in space - the core location at a specific downstream distance fluctuates erratically in time. For example, in the water tunnel described in $\$ 2$, four chords downstream of the hydrofoil the core meanders by $\pm 1 \mathrm{~cm}$. Corsiglia, Schwind \& Chigier (1973) and Baker et al. (1974) believe the vortex meandering is mainly due to free-stream turbulence. This wandering means that any time-averaged Eulerian point measurement is actually a weighted average in both time and space. Baker et al. (1974) have 
measured the root-mean-square displacement of a vortex marked with dye and inferred from this the effect of vortex meandering on their point measurements. They found, in their test facility, that vortex meandering decreased the measured $\left(U_{\theta}\right)_{\max }$ (the maximum tangential velocity around the vortex) to only approximately $70 \%$ of its correct (i.e. no meandering) value, and increased the measured core radius, $R_{\mathrm{c}}$ (the radius at which $U_{\theta}$ is a maximum), by a factor of 2.2 over its correct value. A group at NASA Ames (Chigier \& Corsiglia 1971; Chigier \& Corsiglia 1972) has measured, at different times, tip vortices using two different techniques. In their original work they measured time-averaged point velocities in the trailing vortex. For a particular wing configuration and downstream distance $\left(\alpha=10^{\circ}, x / c=10, x / c\right.$ being the downstream distance from the leading edge normalized by the wing chord.) $\left(U_{\theta}\right)_{\max }$ was found to be 0.15. A year later they refined their measurements (Corsiglia et al. 1973 ) by recording only through-the-core passes made by a flying hot wire, and found $\left(U_{\theta}\right)_{\max }=0.64$ for the same wing configuration. Clearly, averaged pointwise Eulerian measurements are very susceptible to vortex meandering. Their usefulness may be questioned unless the measurements are made within approximately two chords of the wing, where the meandering is small, or unless a conditional sampling technique is used.

The second salient feature of tip vortices in the context of measurement difficulties is their susceptibility to probe interference. Orloff (1971) and Holman \& Moore (1961) have both reported that vortices are sensitive to even very small intrusive probes. For example, the trajectory of trailing vortices produced in the facility used in this experiment is substantially altered when a $2 \mathrm{~mm}$ diameter probe is brought to within $2 \mathrm{~cm}$ of the core. A $0.5 \mathrm{~mm}$ probe directed into the core can produce local vortex breakdown. Measurements taken by previous investigators using pressure probes (e.g. Mason \& Marchman 1972, Logan 1971), vortex meters (e.g. Zalay 1976, Jarvinen 1973), and hot-wire probes (e.g. Corsiglia et al. 1973, Chigier \& Corsiglia 1972, Singh \& Uberoi 1976) are subject to these problems.

The technique employed in the present research - double-pulsed holography of injected buoyant droplets/bubbles in a water tunnel - avoids the vortex meandering and intrusiveness problems discussed previously. It has two additional characteristics which are serendipitously desirable; double-pulsed holography provides an instantaneous picture of the vortex core, when suitable tracers are present, which makes it possible to identify the core structure. It also has the capability to resolve the small scales associated with the vortex core.

\section{Experimental facilities and techniques}

\subsection{Test facility}

Experiments were carried out in the Low Turbulence Water Tunnel (LTWT) at Caltech. The test section of this facility is $30.5 \mathrm{~cm} \times 30.5 \mathrm{~cm}$ and $2.5 \mathrm{~m}$ long. The maximum flow velocity attainable in the test section is $10 \mathrm{~m} / \mathrm{s}$, although for the present research $8 \mathrm{~m} / \mathrm{s}$ was never exceeded in order to avoid trailing vortex cavitation. The free-stream turbulence level of the tunnel is less than $0.05 \%$. A complete description of the facility may be found in Gates (1977).

A rectangular planform NACA 66-209 hydrofoil with no twist was used to generate tip vortices. The hydrofoil design angle of attack is $7.1^{\circ}$. The tip of the foil consisted of a smooth cap with an approximately semicircular cross-section. The chord length, $c$, was $0.152 \mathrm{~m}$ and the distance from the reflection plane horizontal 
(a)

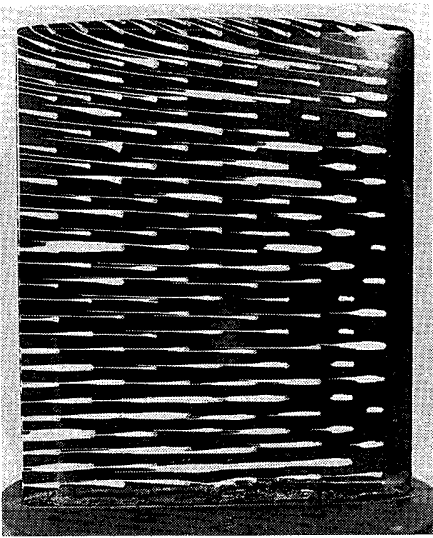

(b)

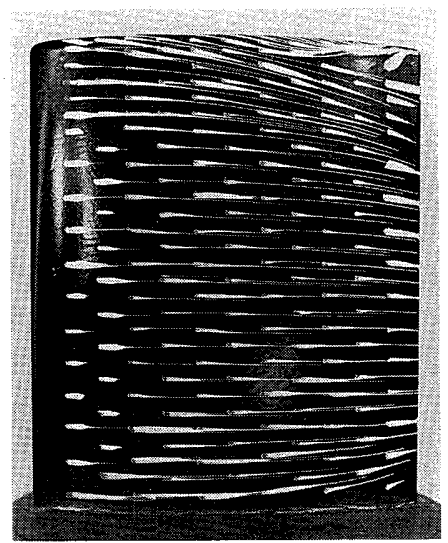

$(c)$

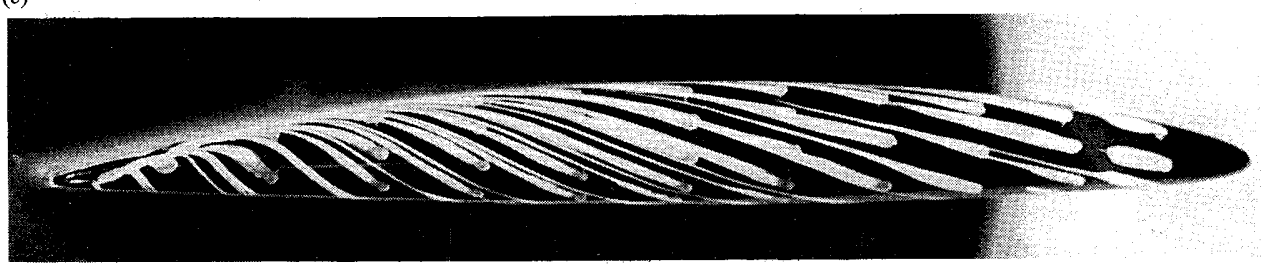

FIGURE 1. Surface flow visualization on the tested hydrofoil at $\alpha=4^{\circ}, R e=1.2 \times 10^{6}$. $(a)$ Pressure side. Flow is right to left. (b) Suction side. Flow is left to right. (c) Inboard view of tip. Flow is right to left.

mount to the base of the tip cap was $0.175 \mathrm{~m}$, which implies an effective aspect ratio of about 2.3. The hydrofoil was mounted in a cylindrical base which was fitted into a $3.1 \mathrm{~cm}$ thick streamlined false floor attached to the true water tunnel floor. The false floor was $51 \mathrm{~cm}$ long. The hydrofoil leading edge was 3 chords downstream of the test section entrance. Figures $1(a)$ and $1(b)$ are photographs of surface flow visualization on the suction and pressure surfaces of the wing at $\alpha=4^{\circ}$. The surface flow visualization was accomplished by removing the hydrofoil from the water tunnel, dotting it with oil-based paint drops, returning the foil to the tunnel, and then quickly accelerating the flow up to a set velocity, causing the drops to be smeared in the local flow direction. (Details of the flow visualization technique are given in Green 1988b.) The photographs show the flow to be virtually twodimensional further than 0.2 chords away from the floor, and therefore that the wingwall interaction only slightly affects the tip flow. These visualizations also showed that over the range of Reynolds numbers studied (roughly $3 \times 10^{5}$ to $1.2 \times 10^{6}$ ), the flow around the hydrofoil is attached for all positive angles of attack less than $12^{\circ}$. Figures $2(a)$ and $2(b)$, for example, show that for $\alpha=10.1^{\circ}$ no separated flow occurs on the hydrofoil, with the exception of a small three-dimensional wing/wall-induced flow separation region confined to within $0.2 c$ of the hydrofoil root.

A $12 \%$ thick, $0.102 \mathrm{~m}$ chord symmetric Joukowski foil with an effective aspect ratio of 3.7 was also tested. This hydrofoil was not fitted with a tip cap; i.e., its tip was squared off normal to the spanwise direction. 
(a)

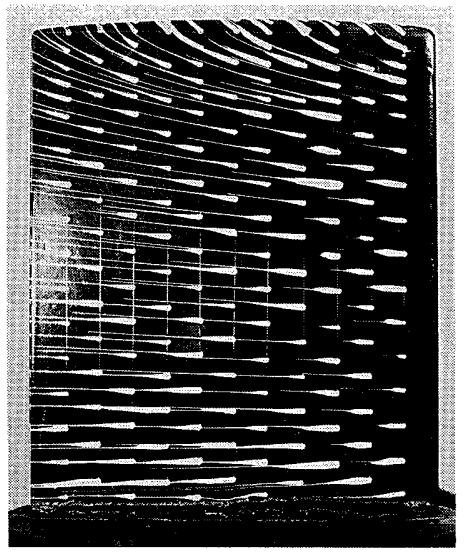

(b)

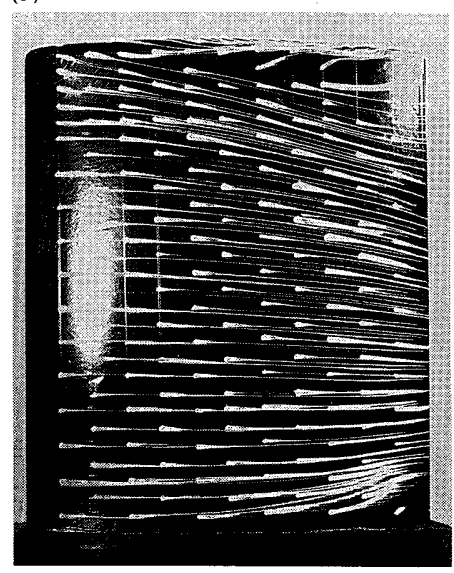

$(c)$

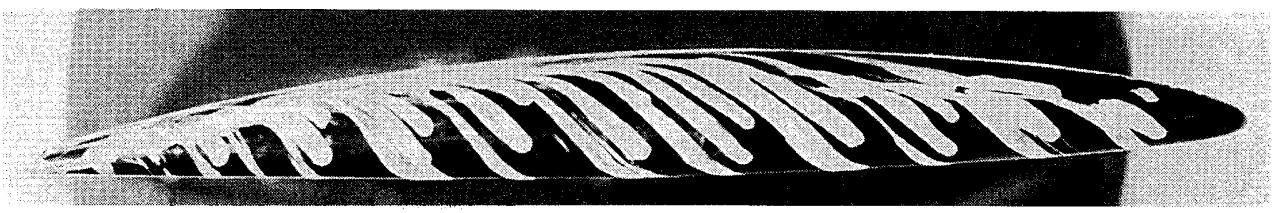

Figure 2. Surface flow visualization on the tested hydrofoil at $\alpha=10.1^{\circ}, R e=1.2 \times 10^{6}$. $(a)$ Pressure side. Flow is right to left. $(b)$ Suction side. Flow is left to right. $(c)$ Inboard view of tip. Flow is right to left.

\subsection{Double-pulsed holographic technique}

Microbubbles, or any other buoyant particles such as oil droplets, may be injected upstream of a hydrofoil mounted in the LTWT, as shown in figure 3 . The microbubbles $(40 \mu \mathrm{m}-400 \mu \mathrm{m}$ in diameter for this study) are particularly useful as nearly Lagrangian flow markers because a net centripetal force slowly drives some of them into the core, where they accurately define the instantaneous vortex centreline location. This feature is important because it permits the instantaneous core location to be identified. The microbubble injector was located 1.5 chords upstream of the hydrofoil, and was positioned laterally such that many bubbles produced by the injector lay on the streamline passing through the vortex centre. The free-stream turbulence in the tunnel and the modest turbulence generated by the injector helped disperse the bubbles over an approximately cylindrical region $5 \mathrm{~cm}$ across. The injector consisted of a $2 \mathrm{~mm}$ diameter glass micropipette heated and pulled to a point, and then broken to yield a nozzle with a $20 \mu \mathrm{m}$ opening. The pipette was connected by means of a $3 \mathrm{~mm}$ stainless steel tube to a compressed air supply. The injector was relocated 20 chords upstream of the hydrofoil for some tests. Results acquired with the injector there confirmed that it was non-intrusive to within experimental error.

A ruby laser with a pulse time of less than $50 \mathrm{~ns}$ duration served as the coherent, collimated holographic light source. Double-pulsed in-line holograms - two holograms of a specified volume taken in quick succession $(150 \mu \mathrm{s}$ or $300 \mu \mathrm{s}$ time separation) - were recorded on holographic film. The flow volume recorded on each hologram is a cylinder $5 \mathrm{~cm}$ in diameter and $30.5 \mathrm{~cm}$ (the tunnel width) long. 


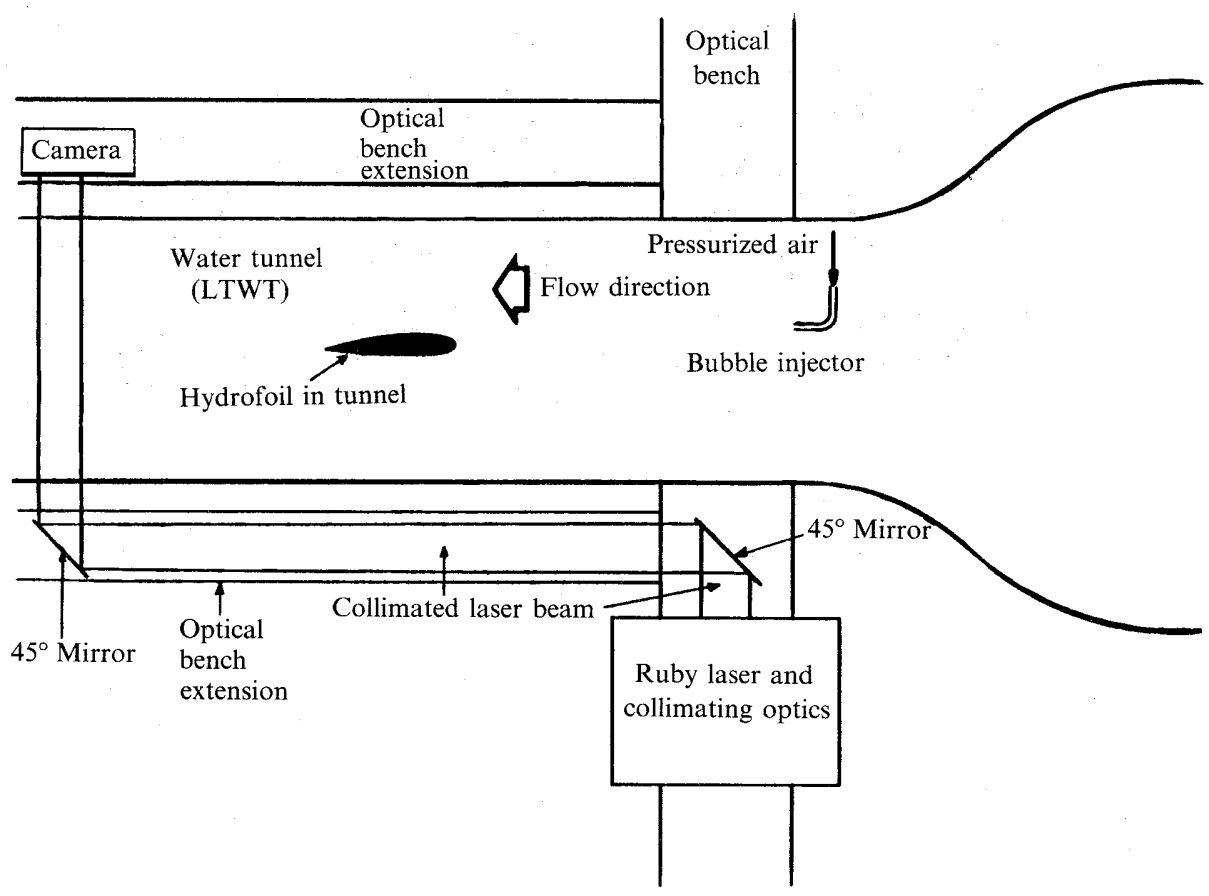

Figure 3. Schematic of double-pulsed holography technique.

After development of the holographic film the holograms were reconstructed by illuminating them with collimated HeNe laser light. Two images were produced by this illumination - a real and a virtual image. The real image was examined by moving the hologram (mounted on an $x-y$-z translation stage) relative to a fixed objective lens. The objective lens in combination with a video camera and monitor produced a $100 \times$ magnified view of the real fluid volume. A factor of 0.91 compression of the real fluid dimension occurred out of the plane of the hologram because of the difference in wavelength of the ruby laser (694 $\mathrm{nm}$ ) and the HeNe laser $(633 \mathrm{~nm})$ light. Allowance was made for this compression in the data reduction. Dimensions in the holographic plane were not affected by the reconstruction laser wavelength.

Figure 4 is a photograph of part of a typical reconstructed double-pulsed hologram. Two images of one bubble in the flow can be seen. The displacement between the two images is proportional to the bubble velocity. In some holograms of the core the bubbles were closely spaced. There was, however, never any difficulty in pairing bubble images because of both the spectrum of bubble sizes, and the differing luminosities of the first and second image of each bubble (caused by the differences in the lasing intensities of the two pulses). In theory, one should be able to obtain complete three-dimensional particle location information from a hologram. However, even on the best holograms, out-of-plane displacements could be measured to only $\pm 130 \mu \mathrm{m}-$ not surprising given the typical bubble size and Fraunhofer holography method used. This shortcoming limited the data to accurate in-plane $\left(U_{x}, U_{y}\right)$ velocities $( \pm 3 \%$ error or better, generally) located accurately in three-dimensional space (refer to figure 5). The $U_{y}$ velocity, in conjunction with precise knowledge of the 


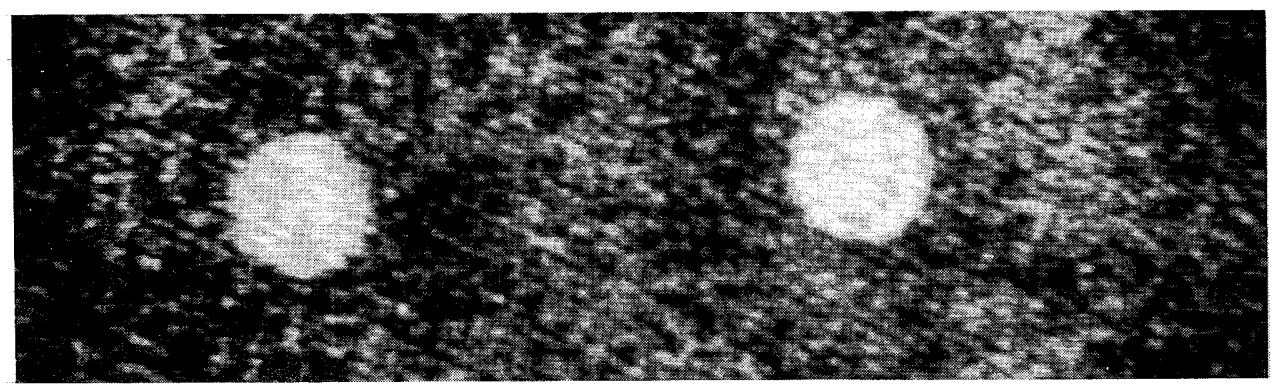

Figure 4. A portion of a typical reconstructed double-pulsed hologram.

The bubble is $160 \mu \mathrm{m}$ in diameter.

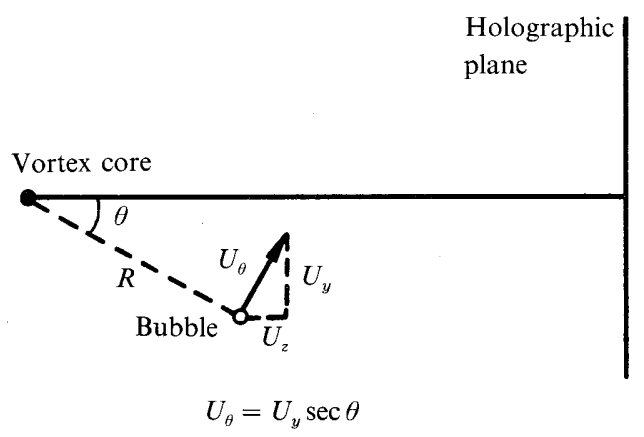

Figure 5. Geometry of tangential velocity measurement.

$U_{x}$ is the velocity component into the page.

instantaneous location of the vortex core, yielded the tangential velocity, $U_{\theta}$, of each bubble about the core. $U_{x}$, the axial component of velocity, was measured directly in the holographic plane to an accuracy of better than 1\%. The error in the out-ofplane displacement can be shown to produce less than a $4 \%$ error in both the radial distance from the centreline $(R)$ and $\theta$ for bubbles at radii greater than $0.02 c$.

Most, but not all, of the tangential velocity data acquired in this way is known to $\pm 4 \%$. Small errors in $U_{y}$, magnified by the sec $\theta$ factor when $\theta$ is near $\pm \pi / 2$, produce large errors in $U_{\theta}$. Restricting data to the sectors $|\theta \pm \pi / 2|>\pi / 6$ effectively eliminates this problem. Radial slip of bubbles into the core could have also contaminated the tangential velocity data by contributing to $U_{y}$. In the first and third quadrants radial slip diminishes $U_{y}$ and hence decreases the inferred $U_{\theta}$; in the second and fourth quadrants the opposite is true. In figure 6 tangential velocity results from the first and third (solid diamonds) and second and fourth (solid boxes) are compared. For $R / c>0.075$ it is apparent that radial slip does not contribute significantly to $U_{\theta}$. Inside $R / c=0.075$ radial slip is important; restricting the accepted data to $|\theta|<\frac{1}{9} \pi$ and $|\theta-\pi|<\frac{1}{9} \pi$ (the open box symbols) in this region ensures that the radial slip does not unduly contaminate the $U_{\theta}$ results.

There are two additional ways to confirm that the effect of bubble slip is slight. One approach is to observe the behaviour of different sized bubbles. In a uniform 


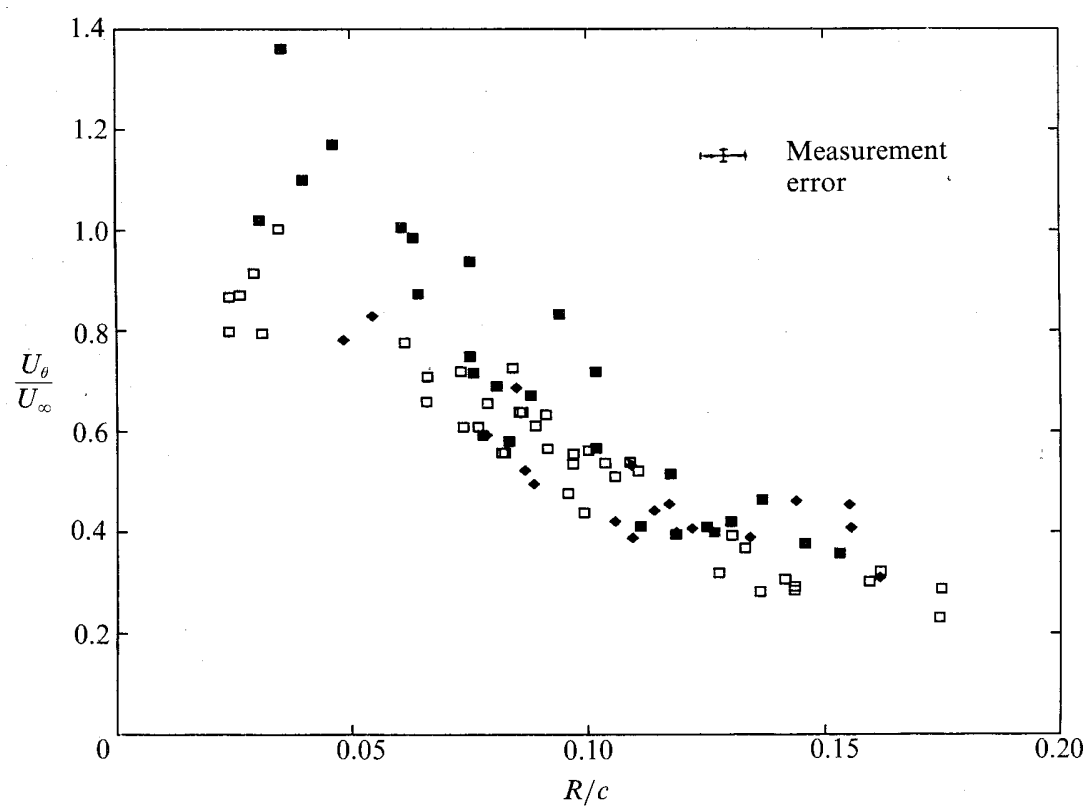

Figure 6. Evidence of radial slip into the trailing vortex. $x / c=2, \alpha=10^{\circ}, R e=6.83 \times 10^{5}$. The open boxes represent data taken in the interval $|\theta|<20^{\circ}$ and $\left|\theta-180^{\circ}\right|<20^{\circ}$. The solid boxes represent $-60^{\circ}<\theta<-30^{\circ}, 120^{\circ}<\theta<150^{\circ}$. The diamonds represent $30^{\circ}<\theta<60^{\circ}$, $-150^{\circ}<\theta<-120^{\circ}$.

pressure gradient of $6.8 \mathrm{MPa} / \mathrm{m} \dagger$, the present best estimate of the maximum radial pressure gradient around any of the observed vortices, the terminal slip velocity of a $100 \mu \mathrm{m}$ diameter bubble is $0.97 \mathrm{~m} / \mathrm{s}$. Doubling the diameter to $200 \mu \mathrm{m}$ almost doubles the slip velocity to $1.77 \mathrm{~m} / \mathrm{s}$. A typical slip velocity for bubbles within $R / c=0.05$ is approximately half this value, perhaps $\approx 0.5 \mathrm{~m} / \mathrm{s}$. At this velocity, bubbles would take $15 \mathrm{~ms}$ to reach the core from $R / c=0.05$. In the same time they would travel downstream a distance $x / c=4.52 \times 0.015 / 0.152=0.4$. This trivial calculation explains the high concentration of bubbles on the vortex centreline, even one chord downstream of the wing trailing edge. Figure 7 shows no significant dependence of $U_{\theta}$ and $U_{x}$ on bubble size, from which we conclude that bubble slip effects are apparently not important.

Small droplets of a heptane-azobenzene mixture were injected in place of air bubbles during one set of tests. These droplets are much less susceptible to slip than air bubbles because the difference in density between the mixture and water is small (about 0.3 difference in specific gravity). The results of measurements made with these injected tracers are documented in Green (1988b), where it is shown that bubble slip effects are unimportant in determining the velocity components. As a final observation, it warrants mention that velocity data acquired from naturally occurring particulates in the LTWT were in agreement with the air bubble data.

$\dagger$ Referring to figure 12, the maximum, time-mean, tangential velocity around the trailing vortex is approximately $0.93 U_{\infty}$, which occurs at $R / c=0.017 \pm 0.005$. For the experimental freestream velocity $(4.52 \mathrm{~m} / \mathrm{s})$, this implies a maximum radial pressure gradient of $\partial p / \partial R=\rho U_{\theta}^{2} / R=$ $6.8 \times 10^{6} \mathrm{~Pa} / \mathrm{m}$, where steady, axisymmetric flow has been assumed in the radial momentum equation. 

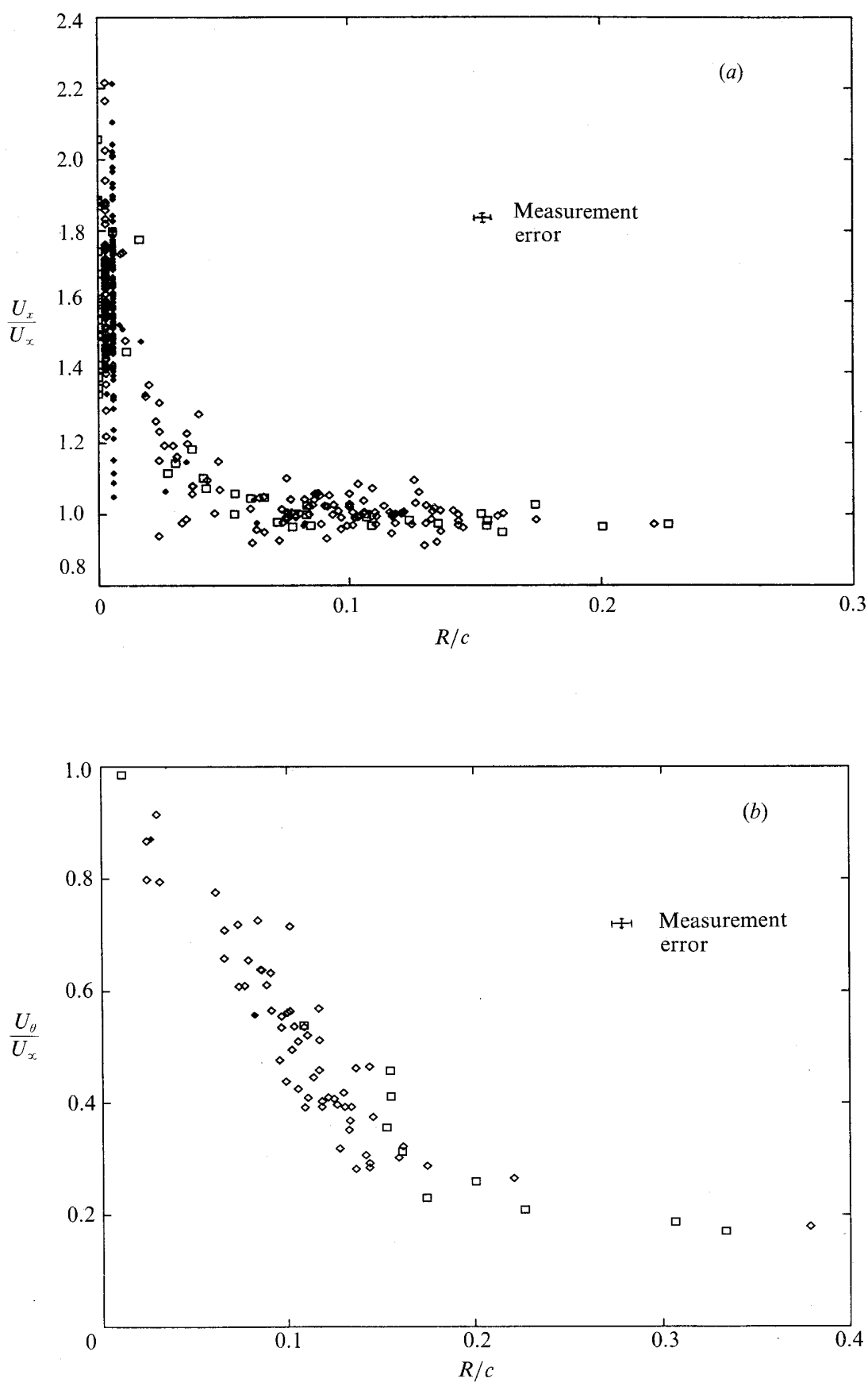

Frgure 7. Evidence of negligible effect of radial slip into the core on the selected data. Same flow conditions as figure 5. The open boxes represent bubbles less than $100 \mu \mathrm{m}$ in diameter. The solid diamonds represent bubbles between $100 \mu \mathrm{m}$ and $200 \mu \mathrm{m}$ in diameter, and the open diamonds represent bubbles larger than $200 \mu \mathrm{m}$. The centreline data have been spread over the region $0 \leqslant R / c \leqslant 0.005$ for clarity. (a) Axial velocity distribution, $(b)$ tangential velocity distribution. 
The concentration of bubbles in the flow was very small. Even on the vortex core itself, bubbles occupied less than $10 \%$ of the core length. Consequently, any influence of the bubbles on the liquid flow must be slight. This statement is borne out by the observation that regions of the flow with very few bubbles had identical mean velocity characteristics to regions with many bubbles.

A few holograms were taken far upstream of the hydrofoil. The mean axial velocity measured holographically matched the free-stream velocity measured independently using a manometer to within $1 \%$. Some of the experiments described in this paper were repeated after water tunnel maintenance and after the hydrofoil rounded tip was replaced. Neither modification affected the results.

\section{Results and discussion}

Only data taken at two downstream distances $(x / c=2$ and 10) and for two angles of attack will be described in detail. A more extensive treatment is given in Green $(1988 b)$. The effect of different tip geometries has been studied and will be described in a future paper.

In the literature some uncertainty surrounds the issue of whether axial velocity excesses or deficits exist in the trailing vortex core. Chigier \& Corsiglia (1971) measured an axial velocity excess in the tip vortex, and Corsiglia et al. (1973) subsequently measured a velocity deficit. Thompson (1975) observed axial velocity deficits for attack angles less than $10^{\circ}$. Both axial velocity excesses and deficits have been measured in the present study - figures $8(a)$ and $8(b)$ are graphic evidence that either may occur. Figure $8(a)$ is a portion of a reconstructed double-pulsed hologram. The out-of-focus bubble pair lies in the vortex core, and has an axial velocity of $1.52 U_{\infty}$, whereas the bubble pair to the left represents a bubble at $R / c=0.18$ travelling at $0.95 U_{\infty}$. Figure $8(b)$ is part of a different reconstructed hologram. The out-of-focus bubble to the right in the core is moving at $0.74 U_{\infty}$ whereas the in-focus bubble at $R / c=0.20$ has an axial speed of $1.03 U_{\infty}$.

\subsection{The trailing vortex near field}

Figure 9 displays the axial velocity distribution $\left(x / c=2, \alpha=10^{\circ}\right)$ around the trailing vortex, as measured from eight double-pulsed holograms. The holograms were taken at random intervals over the course of an hour. The mean axial velocity is equal to the free-stream velocity in the region $R / c>0.03$. The mean axial velocity rises rapidly for $R / c<0.03$, as does the axial velocity unsteadiness. At the centre of the vortex $\overline{U_{x \mathrm{c}}^{*}}=\overline{U_{x \mathrm{c}}} / U_{\infty}$ (the dimensionless mean axial velocity on the vortex centreline) is 1.62. The scatter in the data is a real effect; the experimental error is only $\pm 0.005 U_{\infty}$ for the vast majority of the measurements (see section 3.4$) . \overline{\left(U_{x c}^{\prime}\right)^{*}}$, the dimensionless r.m.s. fluctuating component of the axial velocity on the centreline, is 0.20 . By way of contrast, away from the vortex core the fluctuating component of the velocity is less than $\frac{1}{6}$ of this value $(0.03)$. Clearly, the core axial velocity is highly unsteady. These velocity unsteadiness measurements have been corroborated by independent non-intrusive measurements of core unsteady pressure (Green 1989). (The pressure measurements were made by injecting bubbles upstream of the hydrofoil. The bubbles are driven into the vortex core; they vary their radii in response to the instantaneous local static pressure, analogously to balloons in the atmosphere.) The core unsteadiness is believed to be one cause of the unsteady character of trailing vortex cavitation inception (Green 1988b). 
(a)

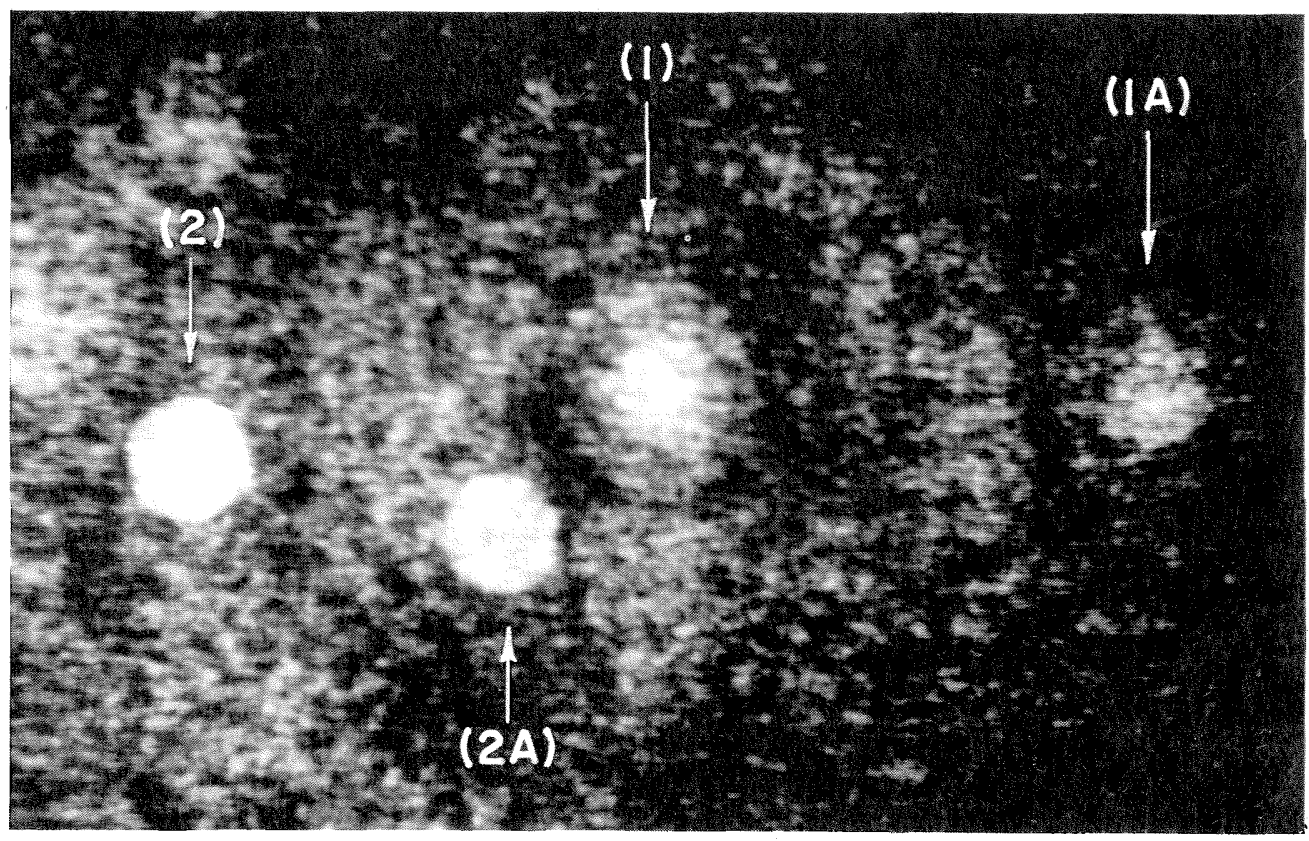

(b)

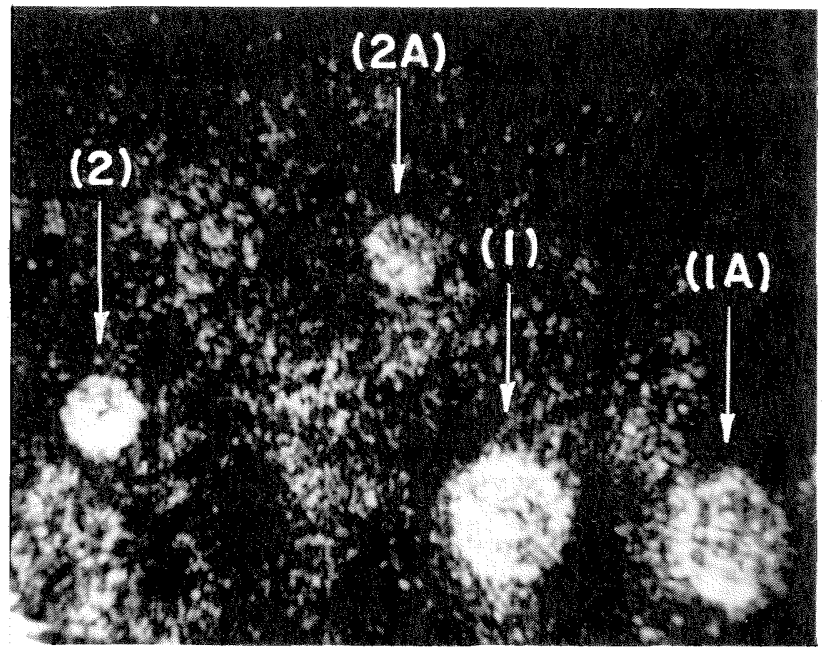

Figure $8(a)$ Example of axial velocity excess. $x / c=2, \alpha=10^{\circ}, \quad R e=5.2 \times 10^{5}$. Scale: $5 \mathrm{~cm} \equiv 0.71 \mathrm{~mm}$ in flow. (b) Example of axial velocity deficit $x / c=10, \alpha=5^{\circ}, R e=6.8 \times 10^{5}$. Scale: $5 \mathrm{~cm} \equiv 1.02 \mathrm{~mm}$ in flow. The two images of the in-core bubble are labelled '1.' The two out-of-core bubble images are labelled ' 2 .' $U_{\infty}$ is left to right.

A careful inspection of the data in figure 9 suggests that $\overline{U_{x \mathrm{c}}^{*}}$ varies from hologram to hologram. The eight holograms plotted in that figure have mean non-dimensional core velocities of $1.493,1.713,1.616,1.654,1.727,1.626,1.569$, and 1.543. A probability plot of the instantaneous core axial velocities acquired from six of the eight holograms is presented in figure 10. The free-stream velocity as measured from 


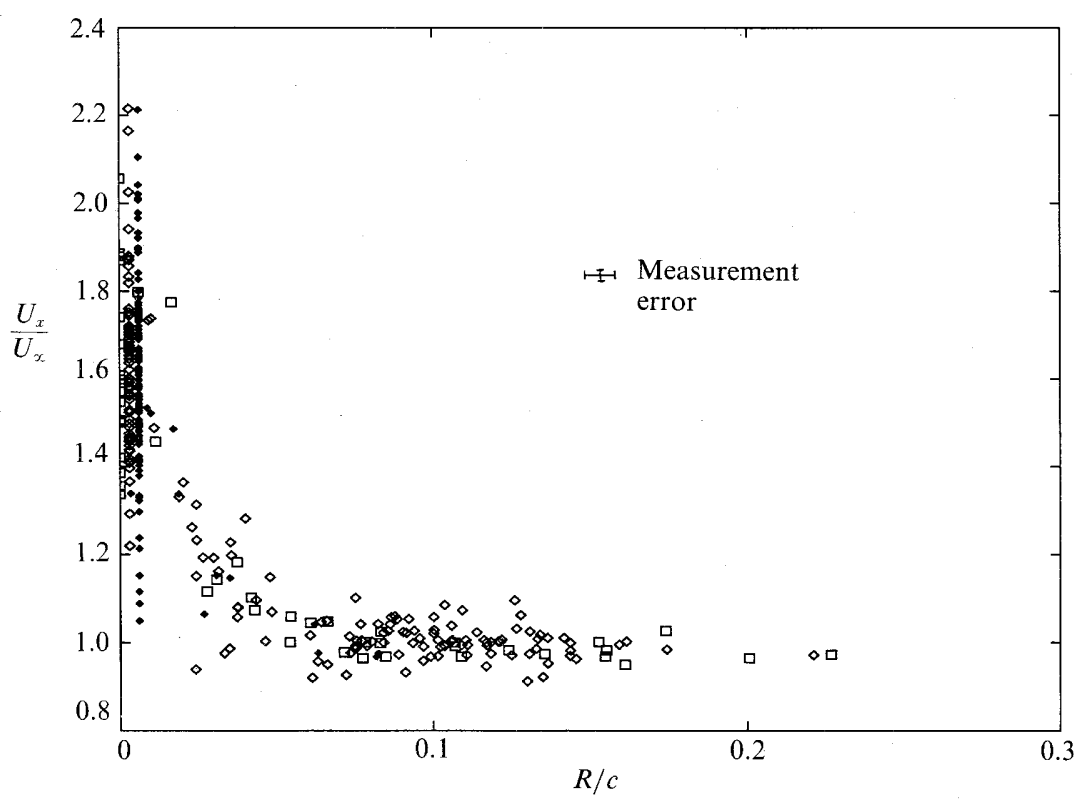

Figure 9. Axial velocity distribution. $x / c=2, \alpha=10^{\circ}, R e=6.83 \times 10^{5}$. The centreline results $(R / c=0)$ have been spread over the region $R / c<0.006$ for clarity. The data acquired from four holograms have been plotted together using a solid square symbol; the data from four other holograms have been plotted using separate symbols.

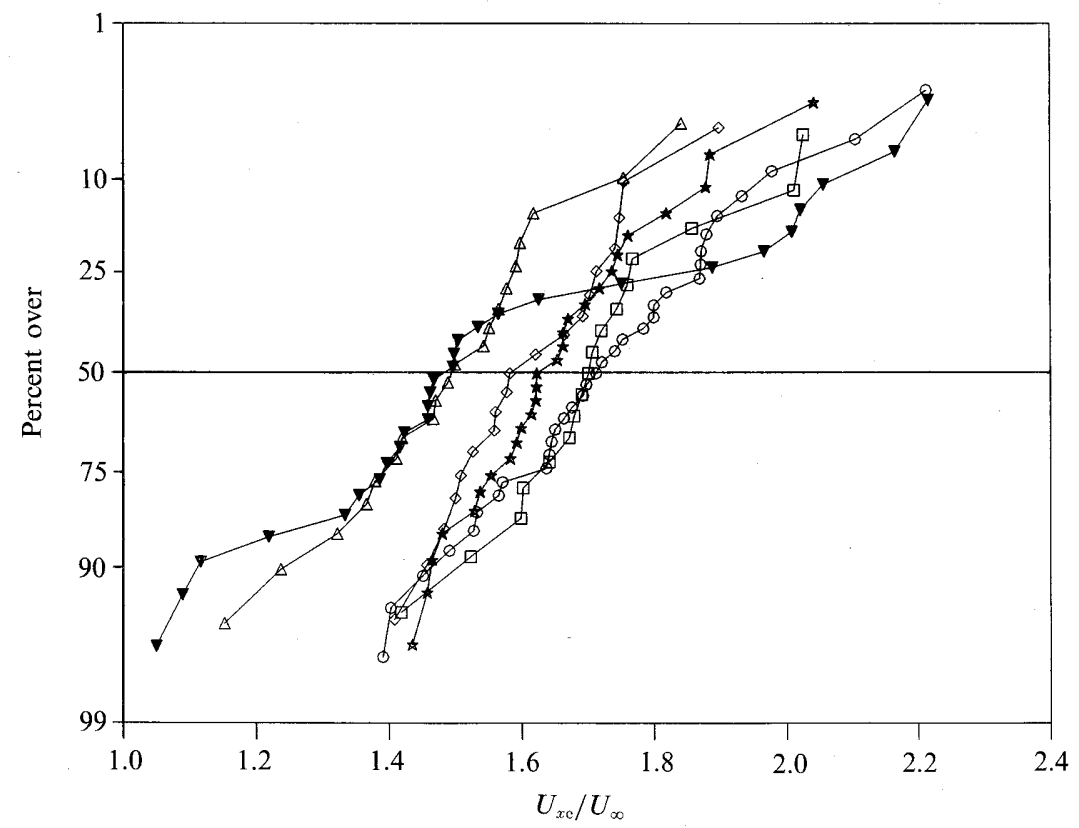

Figure 10. Probability plot of instantaneous centreline axial velocities. $x / c=2, \alpha=10^{\circ}$, $R e=6.83 \times 10^{5}$. Each symbol represents a different hologram. 


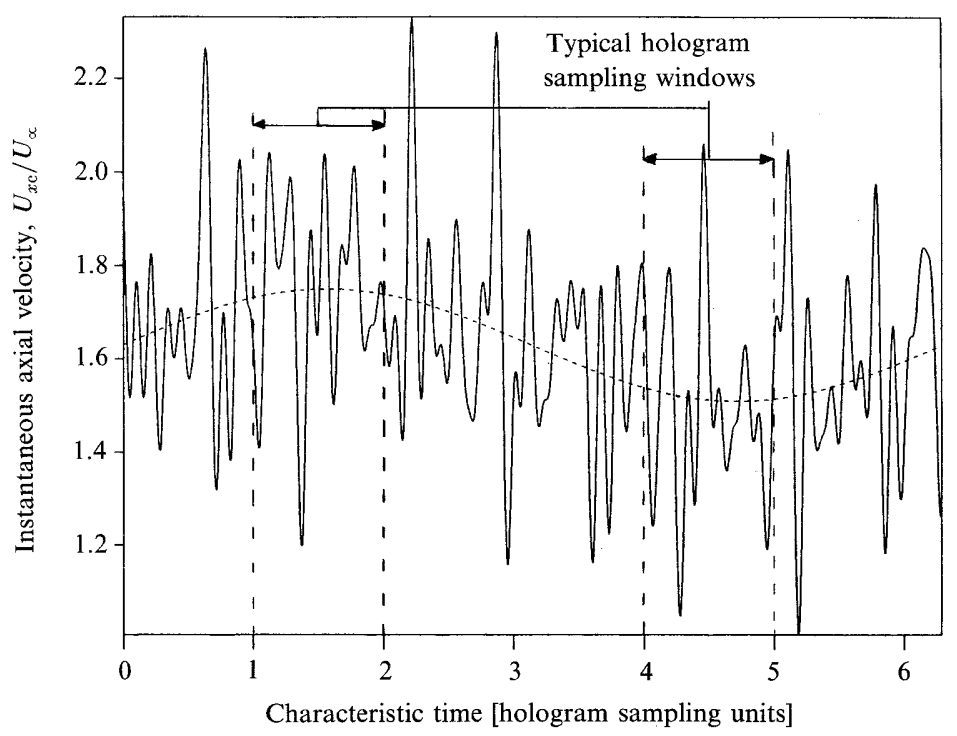

Figure 11. Hypothesized typical time-dependent axial velocity on the vortex centreline showing the 'slow' disturbance. The wavelength of the 'slow' disturbance relative to the hologram sampling length is not known. Each unit of 'characteristic time' is the time required for fluid on the vortex centreline to traverse the $5 \mathrm{~cm}$ width of the hologram.

the holograms varied by less than $1 \%$ during these tests. A 'student-t' test (Kreyszig 1972 ) on the data demonstrates that at the $99.9 \%$ confidence level there is a statistically significant difference between the mean core axial velocities measured at different times. In fact, at the $99 \%$ confidence level there is even a difference between the mean core axial velocity measured at a particular time and that ensemble averaged over many (7) realizations. It should be emphasized at this point that all flow conditions - the angle of attack, the free-stream flow velocity (to within $1 \%$ ) and free-stream turbulence level, and the experimental geometry - were maintained constant during these experiments. It seems highly likely, therefore, that very longwavelength or 'slow' disturbances, with characteristic length large relative to the hologram axial dimension $(\approx 0.3 c)$, propagate along the trailing vortex core. Based on the maximum observed hologram-to-hologram $\overline{U_{x \mathrm{c}}^{*}}$ variation, the authors estimate the peak-to-peak amplitude of the 'slow' disturbance to be $(0.24 \pm 0.06) U_{\infty}$. This slow disturbance amplitude is only approximately $25 \%$ of the measured peak-topeak 'fast' disturbance amplitude, $(1.1 \pm 0.1) U_{\infty}$. Figure 11 shows the hypothesized character of the 'slow' disturbance.

Several vortex core instabilities are known that could be suggested as the cause of these axial disturbances. The Crow (1970) instability is one, but it does not appear to have the correct time constant for the present experiments, nor does the Moore \& Saffman (1973) instability. The swirling flow instability described by Batchelor (1967), is a possible explanation, although the experimental phenomenon requires further study before a firm conclusion may be drawn. The helical mode instability observed by Singh \& Uberoi (1976) is a further possibility, although they observed that instability only for $x / c>13$. The possibility that flow separation over the wing induces this 'slow' disturbance is not congruent with the attached flow shown in figures 1 and 2. 


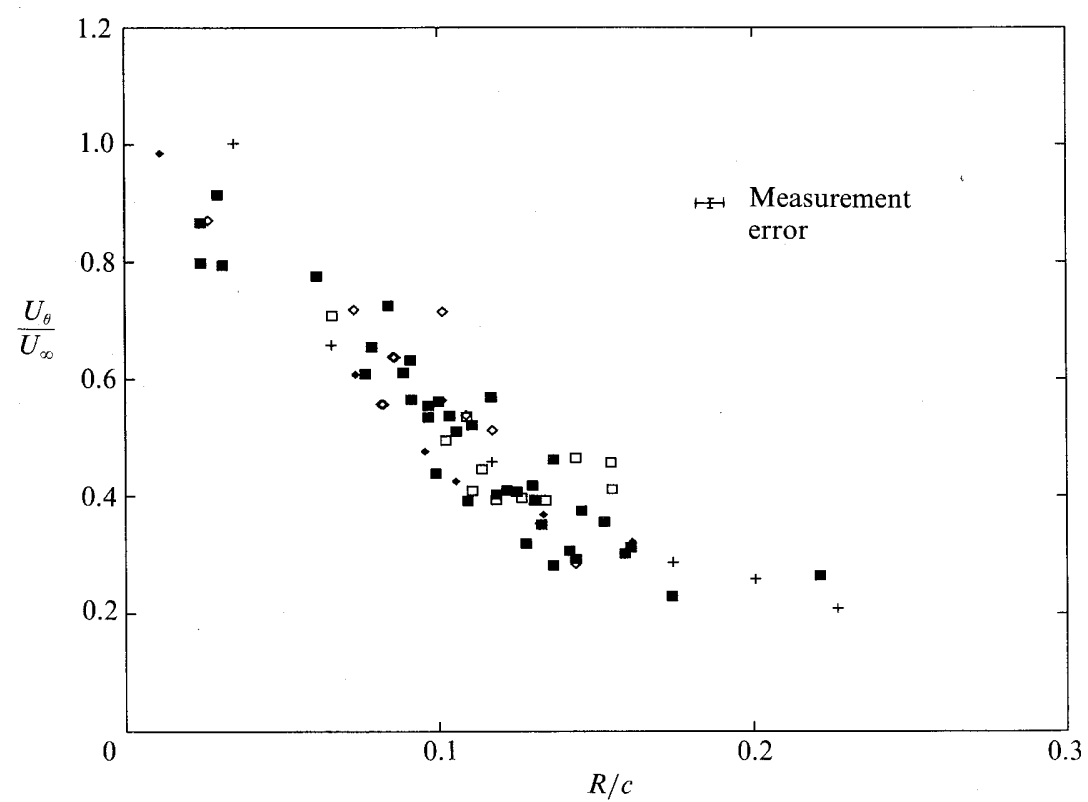

Figure 12. Tangential velocity distribution. $x / c=2, \alpha=10^{\circ}, R e=6.83 \times 10^{5}$. Refer to the caption of figure 9 for an explanation of the symbols.

The tangential velocity distribution measured from the same eight holograms considered in figure 9 is shown in figure 12 . Outside $R / c=0.17$ the data are reasonably correlated by a $1 / R$ variation, but inside this radius the velocity increases less rapidly than $1 / R$. This implies most of the hydrofoil shed vorticity is found in $R / c<0.17$ at a distance only two chords downstream of the hydrofoil leading edge. (Five measurements of $U_{\theta} / U_{\infty}$ were taken in the region $0.3<R / c<0.6$, in addition to the three in $0.17<R / c<0.3$ on figure 12 . For the sake of clarity, they have not been presented in that figure.) The tangential velocity, like the axial velocity, is significantly unsteady. For any particular value of $R / c$ between 0.03 and 0.15 , the spread in $U_{\theta} / U_{\infty}$ is approximately 0.15 ; much larger than the experimental uncertainty of 0.01 , but comparable with the spread in $U_{x} / U_{\infty}$ in the same radius range. The reason why $\overline{U_{x}(R)}$ is equal to $U_{\infty}$ for $R / c>0.03$ although $U_{\theta}(R)$ ceases to be potential for $R / c>0.17$ (which neglecting energy losses on streamlines into the vortex, ensures $U_{x}=U_{\infty}$ ) is not known. The idea that the discrepancy is due to losses along a streamline originating upstream of the hydrofoil is an interesting one because it provides a link between the streamline losses and the tangential velocity distribution.

The data of figure 12 has been replotted in figure 13 with $\theta$ (refer to figure 5 ) as a parameter. No $\theta$-dependence of the tangential velocity is apparent. Similarly, no $\theta$ dependence is apparent in the same data grouped $45^{\circ}$ out-of-phase with the grouping in figure 13 (i.e. $\theta=0^{\circ} \pm 30^{\circ}, 90^{\circ} \pm 30^{\circ}$, etc.). Finally, both at a different angle of attack $\left(\alpha=5^{\circ}\right)$ and at a different downstream distance $\left(x / c=10\right.$ for both $\alpha=5^{\circ}$ and $\alpha=10^{\circ}$ ), no $\theta$-dependence of the tangential velocity has been observed. Thus, in summary, the trailing vortex is axisymmetric to within the accuracy of the experiment. This finding stands in contrast with the asymmetry, observed by Higuchi, Quadrelli \& Farrell (1987) and Stinebring, Farrell \& Billet (1989), of the tip 


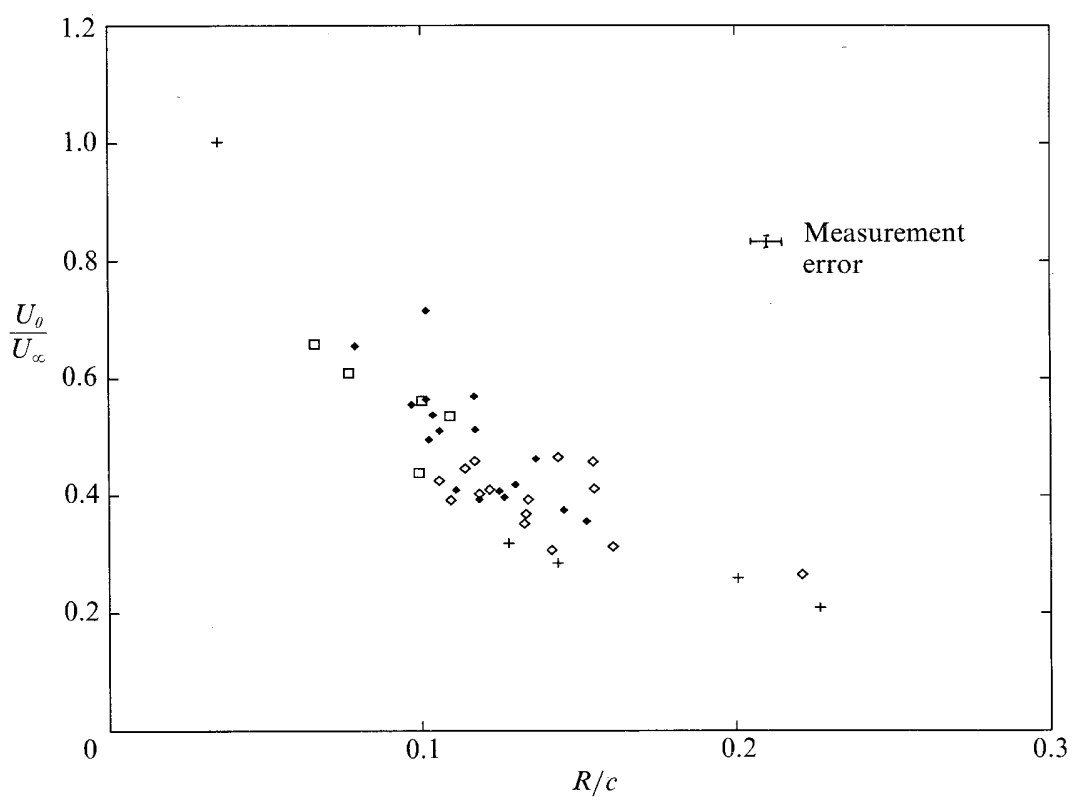

Figure 13. Vortex axisymmetry. $x / c=2, \alpha=10^{\circ}, R e=6.83 \times 10^{5}$. The squares represent bubbles in $\theta=45^{\circ} \pm 30^{\circ}$, the crosses $\theta=135^{\circ} \pm 30^{\circ}$, the open diamonds $\theta=-135^{\circ} \pm 30^{\circ}$, and the solid diamonds $-45^{\circ} \pm 30^{\circ}$. $\theta$ is defined in figure 5 .

vortices generated by respectively an elliptical planform wing and a surface shaped like a propeller blade. Both groups of researchers were examining primarily the very near field of the tip vortex $(x / c<2)$, which may explain the asymmetry of their flows.

The total circulation contained in the trailing vortex was calculated by fitting the measured tangential velocity distribution with a function of the form $U_{\theta}=\Gamma / 2 \pi R$ in the region $R / c>0.17 . \Gamma / c U_{\infty}$ was found to be $0.338\left(\Gamma=0.232 \mathrm{~m}^{2} / \mathrm{s}\right)$ with a variance of 0.017 for a limited sample of 8 bubbles. The bound circulation on a wing is related to the wing lift by $\rho U_{\infty} \Gamma_{\mathrm{B}}=0.5 \rho U_{\infty}^{2} c c_{L}$ ( $c_{L}$ is the wing lift coefficient). Making a correction for the wall effect of the water tunnel (Durand 1963), a finite aspect ratio, thin, elliptical lift distribution wing mounted in the experimental configuration described in $\$ 2$ has:

$$
\frac{\Gamma_{\mathrm{B}}}{c U_{\infty}}=\frac{1.05 \pi\left(\alpha-\alpha_{0}\right)}{1+2 / A} .
$$

The present rectangular planform hydrofoil has a lift coefficient within three percent of the equation (3) value (Thwaites 1960). Equation (3) at $\alpha=10^{\circ}\left(\alpha_{0}=-1^{\circ}\right.$, $A=2.3)$ gives $\Gamma_{\mathrm{B}} / c U_{\infty}=0.339$. This is the same value determined experimentally in the present work. Orloff \& Grant (1973) used a laser doppler velocimeter to measure the tangential velocity distribution around the trailing vortex produced by a rectangular planform wing of aspect ratio 5.3. At a distance eight chords downstream of their wing, they observed that $95 \%$ of the wing bound eirculation was located inside $R / c=0.17$ - in agreement with the present findings.

Hoffmann \& Joubert (1963) have made circulation measurements in a trailing vortex. They found that the turbulent trailing vortex has a region in which the circulation is linear in the logarithm of the radius. However, their logarithmic law is 

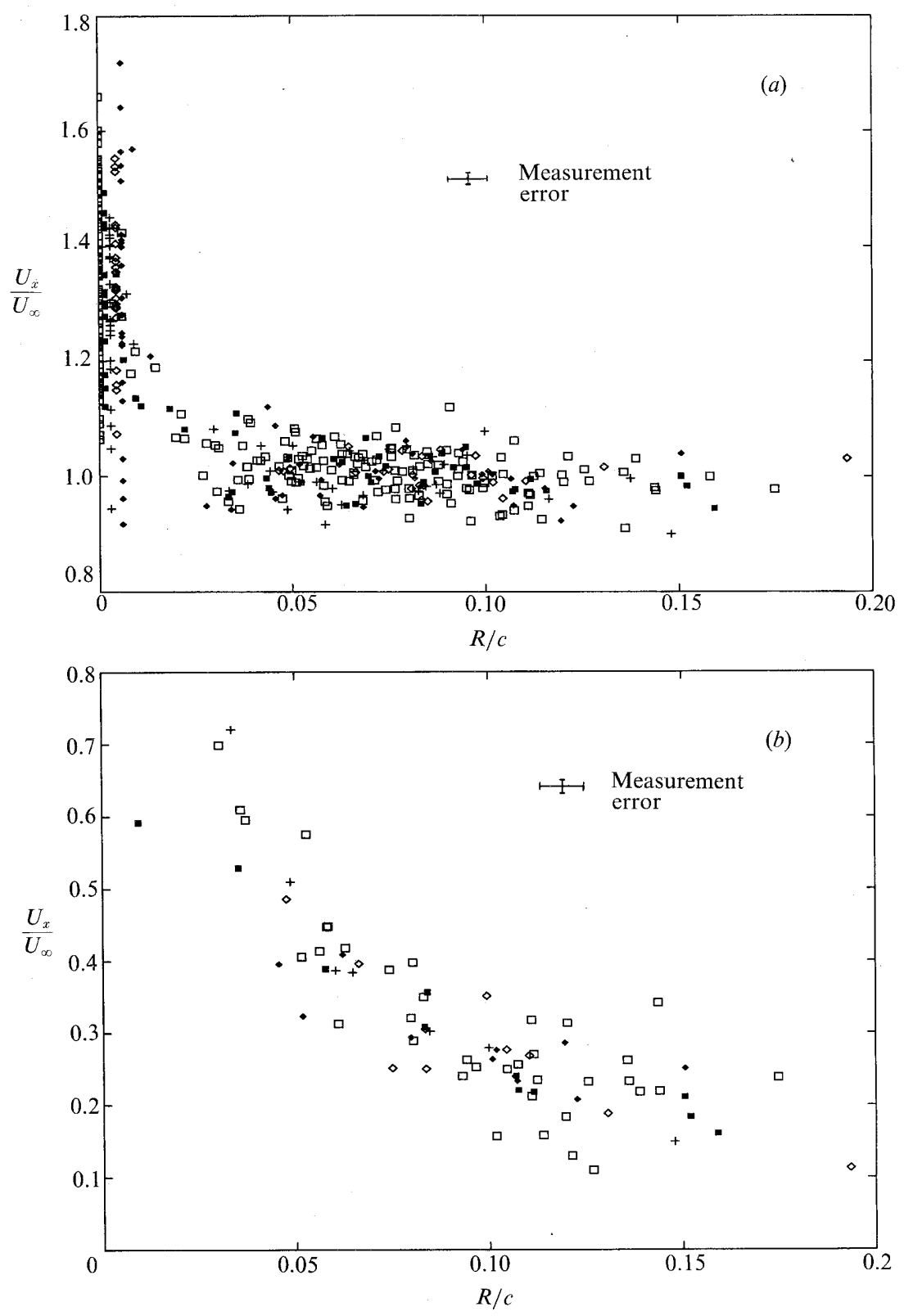

Figure $14(a)$ Axial velocity distribution. $x / c=2, \alpha=5^{\circ}, R e=6.83 \times 10^{5}$. (b) Tangential velocity distribution. $x / c=2, \alpha=5^{\circ}, R e=6.83 \times 10^{5}$.

valid only in the small range $0.008<R / c<0.025$ when expressed in the coordinates of figure 12. Insufficient data has been acquired in that extremely small region to make any comparison with the logarithmic law meaningful.

Figures $14(a)$ and $14(b)$ display the velocity profiles at the same downstream distance and Reynolds number as figures 9 and 12 , but for $\alpha=5^{\circ}$. The axial velocity is equal to $U_{\infty}$ for $R / c>0.025$. The axial velocity mean and fluctuating values rise as $R / c \rightarrow 0$; on the vortex centreline $\overline{U_{x \mathrm{c}}^{*}}=1.33$ and $\overline{\left(U_{x \mathrm{c}}^{\prime}\right)^{*}}=0.16$. At this much smaller angle of attack the centreline axial flow is again highly unsteady. One may 


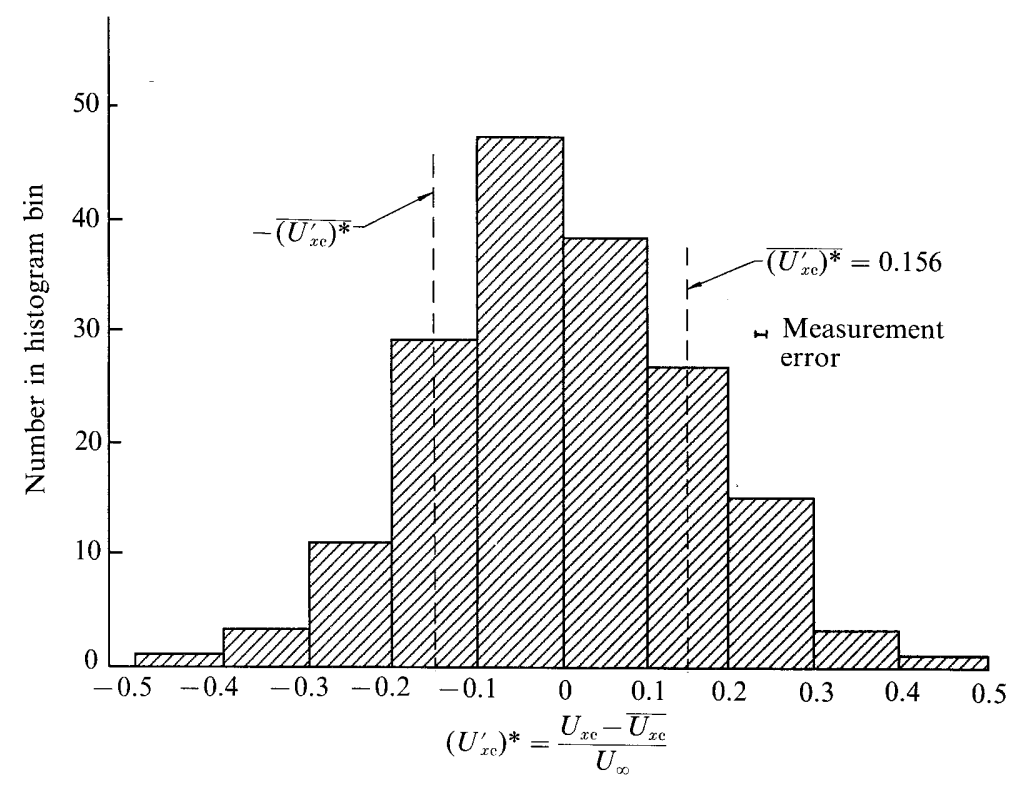

Figure 15. Histogram of instantaneous centreline axial velocities. $x / c=2, \alpha=5^{\circ}, R e=6.83 \times 10^{5}$.

choose to non-dimensionalize the axial velocity on the centreline with the lift coefficient, i.e. $U_{x \mathrm{c}}^{\mathrm{LC}}=\left(U_{x \mathrm{c}}-U_{\infty}\right) /\left(U_{\infty} c_{L}\right)$. Then for $\alpha=10^{\circ}: \overline{U_{x \mathrm{c}}^{\mathrm{LC}}}=0.92, \overline{\left(U_{x \mathrm{c}}^{\mathrm{LC}}\right)^{\prime}}=0.30$ whereas for $\alpha=5^{\circ}: U_{x \mathrm{c}}^{\mathrm{LC}}=0.89,\left(U_{x \mathrm{c}}^{\mathrm{LC}}\right)^{\prime}=0.43$. The good agreement between the two values of $\overline{U_{x \mathrm{c}}^{\mathrm{LC}}}$ makes it plausible that the axial velocity excess in the near field trailing vortex is proportional to the absolute value of the wing bound circulation. The unsteadiness in axial velocity is not proportional to the lift coefficient. It would be interesting to pursue these observations by making further measurements at different angles of attack.

There is no evidence of low frequency core axial velocity disturbances at this angle of attack $\left(\overline{U_{x \mathrm{c}}^{*}}\right.$ is $1.31,1.28,1.34,1.33,1.33,1.38,1.35$, and 1.31 for the eight holograms). The small hologram-to-hologram variations in mean centreline velocity are well within the expected range of fluctuations caused by sampling from a population with a high variance. The reason for the absence of low frequency disturbances at this angle of attack is not known. An examination of the $\dot{\theta}$ dependence of tangential velocity, analogous to that of figure 13 , reveals that for $\alpha=5^{\circ}$ the trailing vortex is also axisymmetric. Figure 15 is a histogram of axial velocities in the trailing vortex core, which shows the unsteady axial velocity to be normally distributed.

Several authors have tried to predict the axial flow in trailing vortices analytically. Batchelor (1964) has analysed trailing vortex flow by considering the Bernoulli equation on a streamline, which originates upstream of a wing where the pressure and velocity are respectively $U_{\infty}$ and $p_{\infty}$, and which passes into the axisymmetric vortex core. The axial velocity in the trailing vortex is then:

$$
U_{x}^{2}(R)=U_{\infty}^{2}-U_{\theta}^{2}+2 \int_{r=R}^{\infty} \frac{U_{\theta}^{2}}{r} \mathrm{~d} r-2 \Delta H
$$


where $\Delta H$ is the total specific energy loss between the two points on the streamline. In the centre of the trailing vortex, equation (1) becomes:

$$
U_{x \mathrm{c}}^{*}=\frac{U_{x \mathrm{c}}}{U_{\infty}}=\left[1+p_{\mathrm{c}}^{*}-\frac{2 \Delta H}{U_{\infty}^{2}}\right]^{\frac{1}{2}}
$$

where $p_{c}^{*}$ is the free-stream to core pressure drop normalized by the free-stream dynamic pressure. The steady, axisymmetrical radial momentum equation may be used in conjunction with figure 12 to find $p_{\mathrm{c}}^{*}=4.4 \pm 0.4$. Green (1989) has also measured the dimensionless core pressure directly and found $p_{\mathrm{c}}^{*}=3.3$. If we accept an intermediate value of 3.8 as correct, equation (2) implies $2 \Delta H / U_{\infty}^{2}=2.2$ (to ensure $\left.\overline{U_{x \mathrm{c}}^{*}}=1.62\right)$. Clearly, $\Delta H$ is significantly large, in contrast with the assumption implicit in many of Batchelor's remarks. (Note that setting $\Delta H=0$ with $\overline{U_{x \mathrm{c}}^{*}}=1.62$ implies $p_{\mathrm{c}}^{*}=1.63$, which does not match the measured value to within a factor of 2.) Moore \& Saffman (1973) and Saffman (1973) analyse axial flow in laminar and turbulent trailing vortices respectively, but they are limited to lightly loaded wings and small axial velocity perturbations from the free-stream, and thus do not really apply to the present experimental situation.

\subsection{The trailing vortex far field}

This subsection is concerned with measurements taken ten chords downstream of the hydrofoil leading edge, a distance much larger than that at which, according to Spreiter \& Sacks (1951), the trailing vortex sheet has fully rolled up. Figure 16(a) is a plot of the axial velocity distribution at $x / c=10$ with the same flow conditions as figure 9. Four holograms, differentiated in the figure by four different symbols, were analysed to yield these results. $\overline{U_{x \mathrm{c}}^{*}}$ is considerably less than the value at $x / c=2$ $\left(1.12 U_{\infty}\right.$ compared with $\left.1.62 U_{\infty}\right)$, though it is still elevated above $U_{\infty}$, and the core flow is still highly unsteady: $\frac{\infty}{\left(U_{x \mathrm{c}}^{\prime}\right)^{*}}=0.18 . \overline{U_{x}}$ differs significantly from $U_{\infty}$ only in $R / c \leqslant 0.04$; apparently this characteristic core dimension is fairly invariant with downstream distance. A histogram of centreline velocities, which is not presented here, shows the axial velocity again to be normally distributed. Low-frequency axial velocity disturbances are also observed at this value of $x / c$.

The mean tangential velocity distribution, figure $16(b)$, matches that at $x / c=2$ very well, with only perhaps a slight elevation of $U_{\theta}$ above the $x / c=2$ value in the region $0.15<R / c<0.25$. However, the unsteadiness in tangential velocity is significantly less at this larger downstream distance. The circulation around the trailing vortex is virtually identical to that at $x / c=2 ; \Gamma / c U_{\infty}=0.342$ with a variance of 0.003 (33 data points). This is in agreement with the observation that for $x / c \geqslant 2$ virtually all the foil bound vorticity is located in a circle of radius $0.17 c$.

The tangential velocity data establish an upper bound on the vortex core growth between $x / c=2$ and $x / c=10$, which may be compared to the simple Lamb (laminar) vortex model. The radius of a Lamb vortex grows like $(4 v t)^{\frac{1}{2}}$. Using the convection time between $x / c=2$ and $x / c=10$ in this expression, one can show that an approximate upper bound on the effective kinematic viscosity in the core is $\nu_{\text {eff }}=35 \nu$, which is fairly low in view of the highly unsteady core flow. It is similarly surprising that the axial velocity varies strongly with downstream distance despite only slight variations in tangential velocity.

Figure $17(a)$ shows the axial velocity distribution at $x / c=10, \alpha=5^{\circ}$. The axial velocity excess of figure 14 has here become a pronounced velocity deficit $\left(\overline{U_{x \mathrm{c}}^{*}}=\right.$ $0.705)$. The 'axial-core' radius at which $U_{x}$ differs from $U_{\infty}$ is approximately $0.04 R / c$. The core unsteadiness is considerably less than at $\left.x / c=2\left(\overline{U_{x c}^{\prime}}\right)^{*}=0.067\right)$, and the 

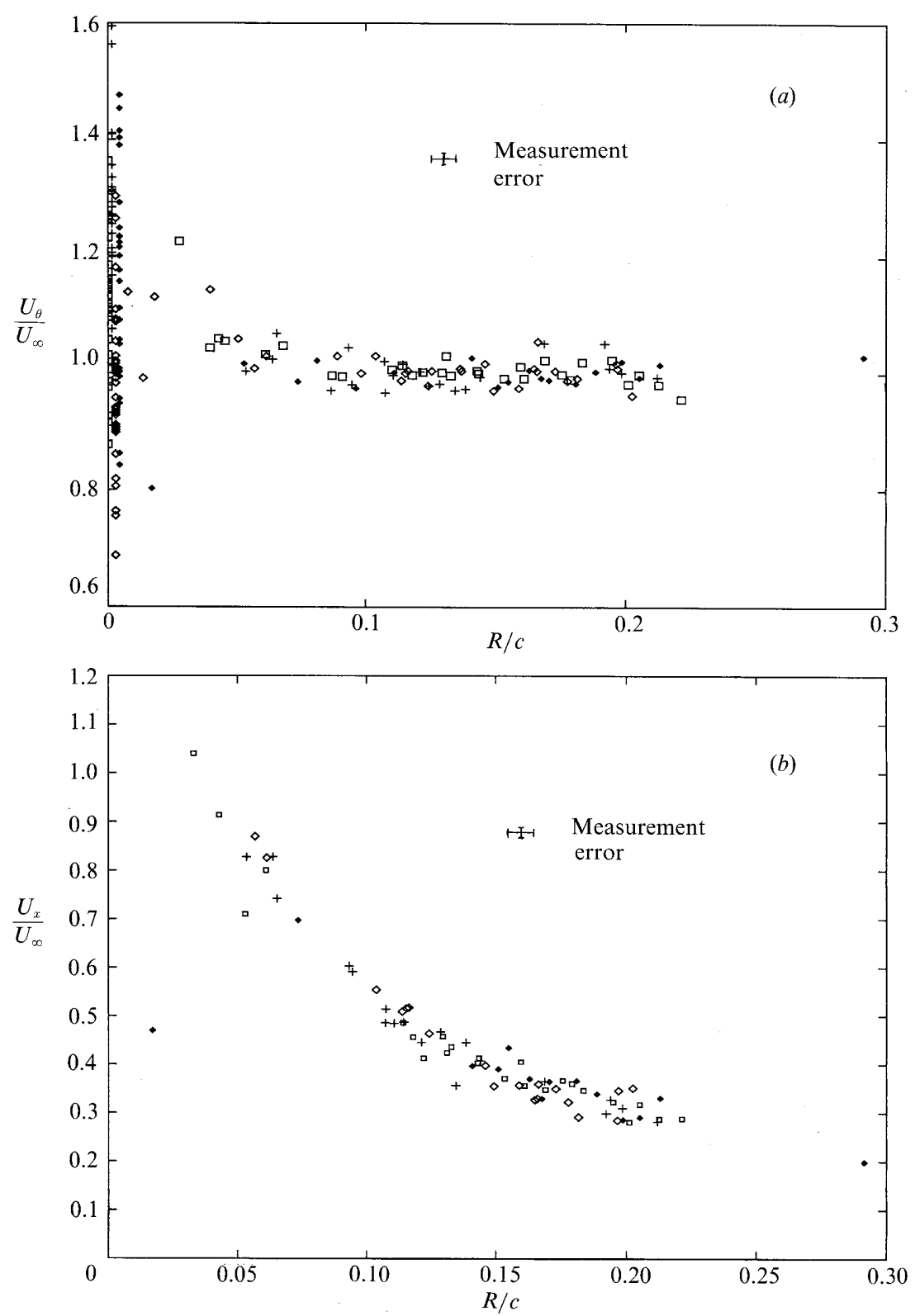

Figure $16(a)$ Axial velocity distribution. $x / c=10, \alpha=10^{\circ}, R e=6.83 \times 10^{5}$. (b) Tangential velocity distribution. $x / c=10, \alpha=10^{\circ}, \operatorname{Re}=6.83 \times 10^{5}$.

unsteady instantaneous velocity is still normally distributed. No low frequency axial disturbances exist. Both the $\alpha=5^{\circ}$ and $\alpha=10^{\circ}$ results show the centreline axial velocity decreases as $x / c$ increases. Similar behaviour is observed with the Joukowski foil, for which $\overline{U_{x \mathrm{c}}^{*}}$ decreases from 1.17 to 1.04 to 0.96 as $x / c$ increases from 2 to 6 to $15\left(\alpha=6.2^{\circ}\right.$ and $\left.R e=4.1 \times 10^{5}\right)$. Non-dimensionalization of axial velocity with the wing lift coefficient, as discussed in section 3.1, shows that in the far field the axial velocity distribution is not proportional to $c_{L}$. 

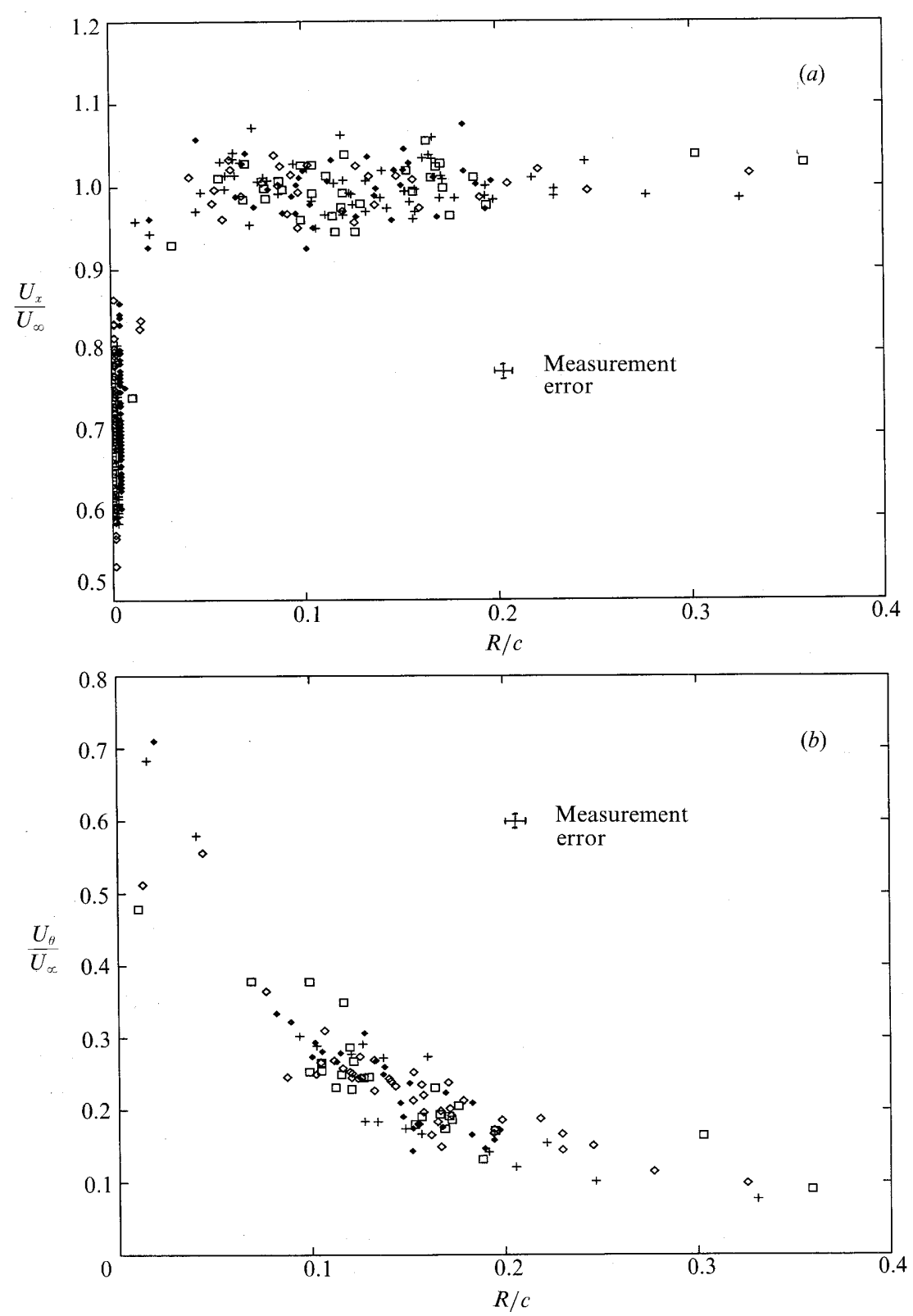

Figure $17(a)$ Axial velocity distribution. $x / c=10, \alpha=5^{\circ}, R e=6.83 \times 10^{5} .(b)$ Tangential velocity distribution. $x / c=10, \alpha=5^{\circ}, R e=6.83 \times 10^{5}$.

The mean tangential velocity data (refer to figure $17(b)$ ) are virtually indistinguishable from that further upstream, shown in figure $14(b)$, although the tangential velocity fluctuations are, as for the $\alpha=10^{\circ}$ case, much smaller. Apparently the tangential velocity distribution varies extremely slowly with $x / c$. The circulation outside $R / c=0.17$ is $\Gamma=0.129 \mathrm{~m}^{2} / \mathrm{s}$ with a variance of 0.003 for a sample of 39 bubbles. Thus $\Gamma / c U_{\infty}=0.188$. This is in very good agreement with equation (3), 
$\Gamma_{\mathrm{B}} / c U_{\infty}=0.185$, which further substantiates the claim that the trailing vortex circulation is equal to the bound circulation of the wing.

The 'overshoot of circulation,' predicted by Govindaraju \& Saffman (1971) to occur at large downstream distances, has not been observed. Phillips (1981) has given theoretical reasons why this circulation overshoot has never been observed around real trailing vortices. Phillips (1981) has also predicted on theoretical grounds that a lightly elliptically loaded wing should produce a trailing vortex whose maximum tangential velocity falls like $(x / c)^{-\frac{1}{4}}$. This fairly rapid decay of tangential velocity has not been observed in this experiment.

\subsection{Reynolds-number effects}

All of the data presented to this point were taken at a fixed Reynolds number of $6.83 \times 10^{5}$. Figure 18 is an attempt to study Reynolds-number effects by plotting the mean vortex centreline axial velocity on axial distance versus Reynolds-number coordinates. Many of the individual points on this plot represent the results of only one hologram (i.e. the flow imaged at one instant in time only), and, because of the highly unsteady flow, may be considerably in error. The expected error is about $5 \%$ in $\overline{U_{x c}^{*}}$ for each point. Nonetheless, several trends are clear. For $x / c=2$ there is no discernible variation of $\overline{U_{x c}^{*}}$ with Reynolds number. This behaviour is also observable at an attack angle of $10^{\circ} ; \overline{U_{x \mathrm{c}}^{*}}$ varies by less than $5 \%$ (from 1.59) as the Reynolds number is varied from $1.5 \times 10^{5}$ to $7.7 \times 10^{5}$ at $x / c=2$. By way of contrast, for $x / c \geqslant 3$ there is a considerable Re-dependency. At any particular value of $x / c$ in this range, $\overline{U_{x \mathrm{c}}^{*}}$ is roughly Gaussian in shape, rising to a maximum at $R e \approx 6 \times 10^{5}$ and falling significantly away from this Reynolds number. The Joukowski foil trailing vortex is also strongly $R e$-dependent. At $x / c=6$ for $\alpha=6.2^{\circ}, \overline{U_{x \mathrm{c}}^{*}}$ rises monotonically from 0.82 to 0.94 to 1.07 as the Reynolds number is increased from $1.0 \times 10^{5}$ to $2.2 \times 10^{5}$ to $7.8 \times 10^{5}$. It has been suggested that the Reynolds number dependence results from boundary layer transition on the hydrofoil, but this hypothesis does not explain the absence of $R e$-dependence at small downstream distances, nor does it seem to be consistent with the $R e$-independence of the mean tangential velocity profile.

The r.m.s. fluctuating centreline velocity is displayed in figure 19 as a function of

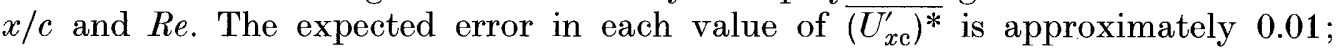
broadly, the core unsteadiness diminishes as $x / c$ increases. For intermediate values of $x / c$ ( 3 or 4 , say) there is a significant Reynolds number effect. At larger downstream distances this dependence is less apparent. The linear regression best fit of $\overline{\left(U_{x \mathrm{c}}^{\prime}\right)^{*}}$ against $\overline{U_{x \mathrm{c}}^{*}}$ has a slope of 0.14 , although the correlation coefficient is only 0.77 . The implication is that, generally, greater unsteadiness in the core is associated with larger dimensionless mean core velocities, though there are likely other factors

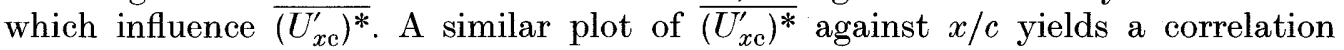
coefficient of only 0.70 - less than that of the aforementioned plot. The mean core axial velocity is thus related to $\overline{\left(U_{x c}^{\prime}\right)^{*}}$ more strongly than simply by the dependence of both $\overline{\left(U_{x \mathrm{c}}^{\prime}\right)^{*}}$ and $\overline{U_{x \mathrm{c}}^{*}}$ on downstream distance. It seems possible, therefore, that the axial unsteadiness is caused by the unstable interaction of the swirling flow with the axial velocity profile.

\subsection{Vortex core observations}

Double-pulsed holograms of buoyant particles contain information not only about local velocities, as described in $\S 1$, but also about global flow structure. In addition, air bubbles are readily deformed and some information about pressure gradients 


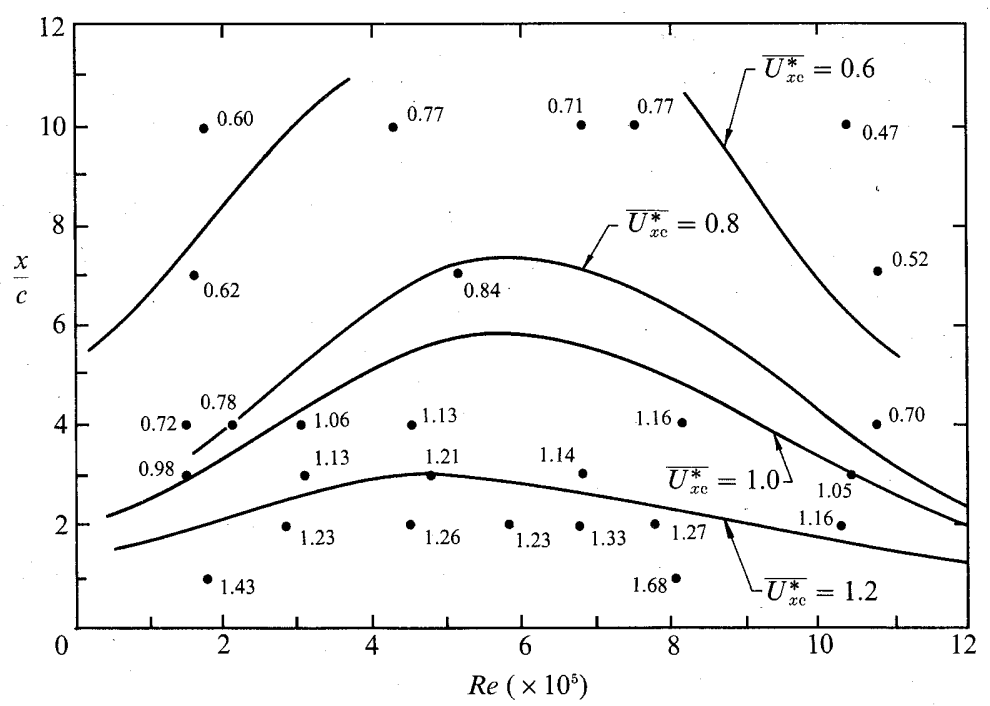

Figure 18. Mean centreline axial velocity, $\alpha=5^{\circ}$. The uncertainty in each measurement of $\overline{U_{x \mathrm{c}}^{*}}$ is $\pm 5 \%$.

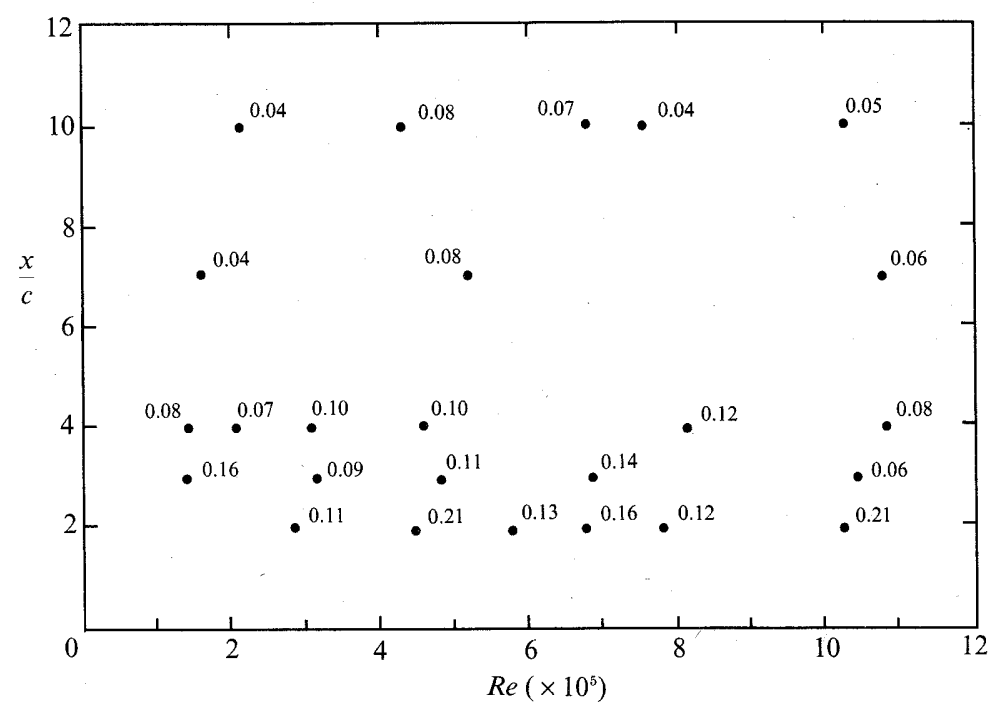

FtgURe 19. Fluctuating centreline axial velocity, $\alpha=5^{\circ}$. The uncertainty in each measurement of $\left(U_{x \mathrm{c}}^{\prime}\right)^{*}$ is \pm 0.01 .

and/or shearing stresses may be derived from their appearance. This subsection documents these qualitative results.

One feature of all the trailing vortices studied is the deformed appearance of bubbles close to, but not immediately on, the vortex centreline. Figures $20(a)$ and $20(b)$ are two reconstructed double-pulsed holograms of bubbles near the radius at which $U_{\theta}$ is maximal. The bubbles' major to minor axes are in the ratio of 3 to 2 . We do not know if these deformations are due to large radial pressure gradients or axial 
(a)

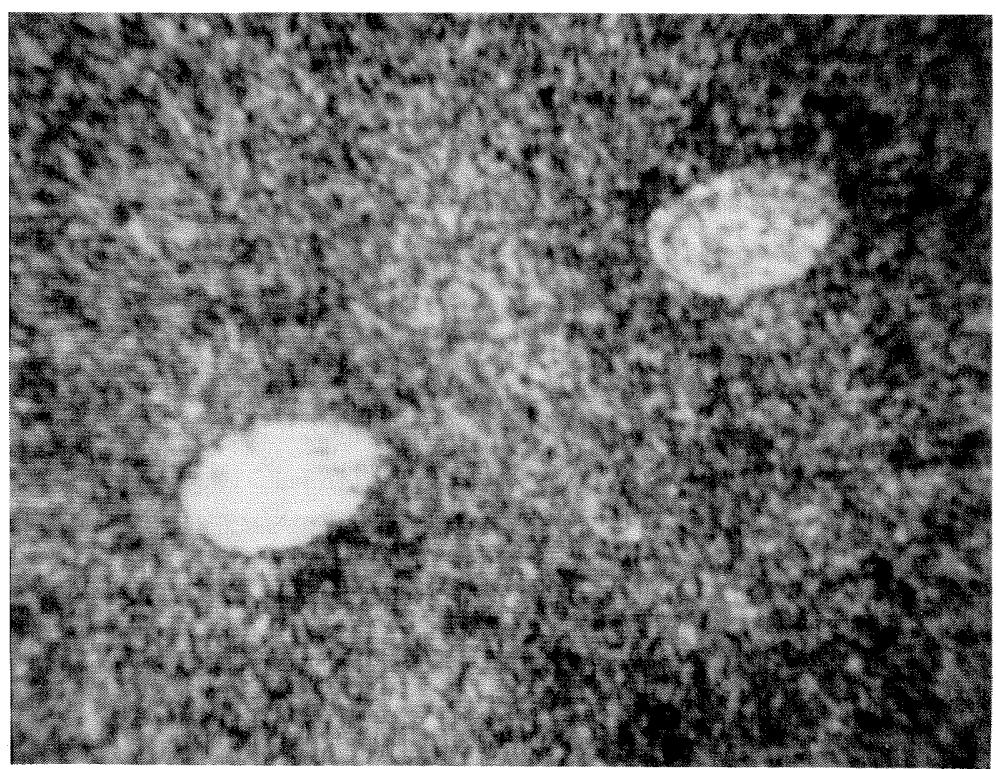

(b)

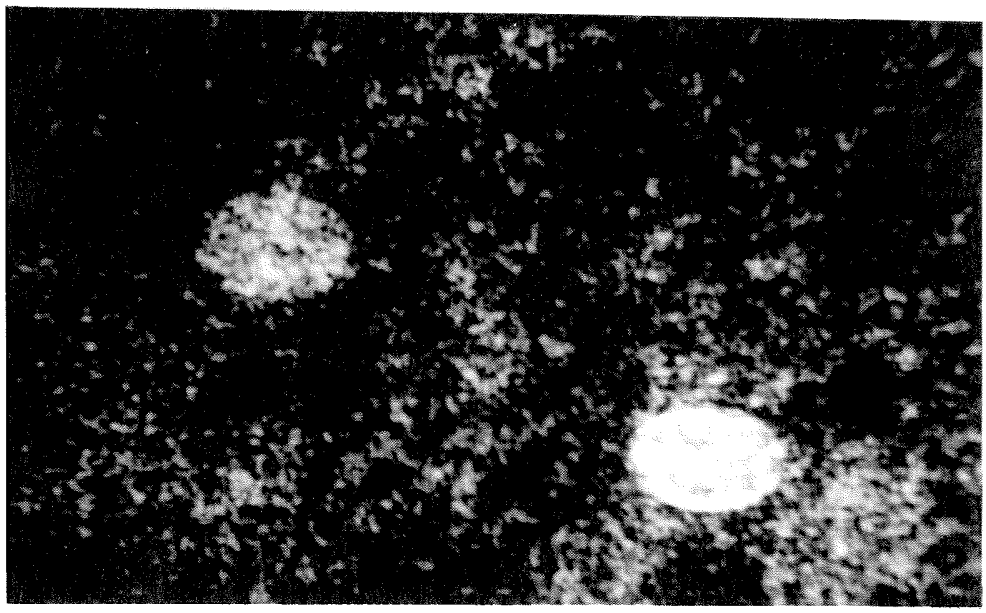

Figure 20. Double-pulsed hologram of bubble near core. (a) $x / c=2, \alpha=5^{\circ}, R e=6.83 \times 10^{5}$. The bubble is located at $R / c=0.033$. Scale: $5 \mathrm{~cm} \equiv 0.68 \mathrm{~mm}$ in flow. $(b) x / c=2, \alpha=10^{\circ}$, $R e=6.83 \times 10^{5}$. The bubble is located at $R / c=0.025 .5 \mathrm{~cm} \equiv 0.78 \mathrm{~mm}$ in flow. $U_{\infty}$ is left to right.

shear stresses. The bubble deformations are more extreme for bubbles closer to the vortex centreline - the off-centreline bubble in figure $21(a)$ (at $R / c \approx 0.011$ ) is roughly twice as long as it is wide, as are the bubbles in figure $21(b)(R / c \approx 0.004)$ and figure $21(c)(R / c \approx 0.003)$. Precisely on the vortex centreline most of the bubbles are very nearly spherical. The few deformed bubbles on the centreline are either very large (and probably cavitating - these bubbles were excluded from the velocity statistics), or else part of one of the core structures described below. The severe deformation of bubbles near the vortex centreline may be an important feature of the cavitation inception process. 
(a)

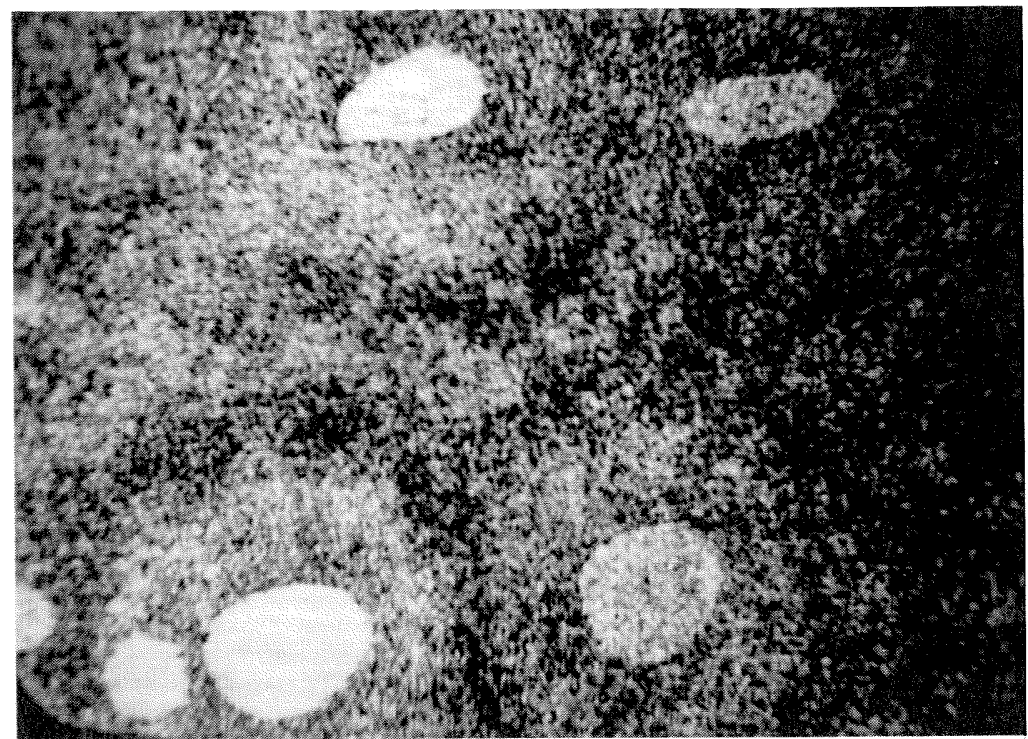

(b)

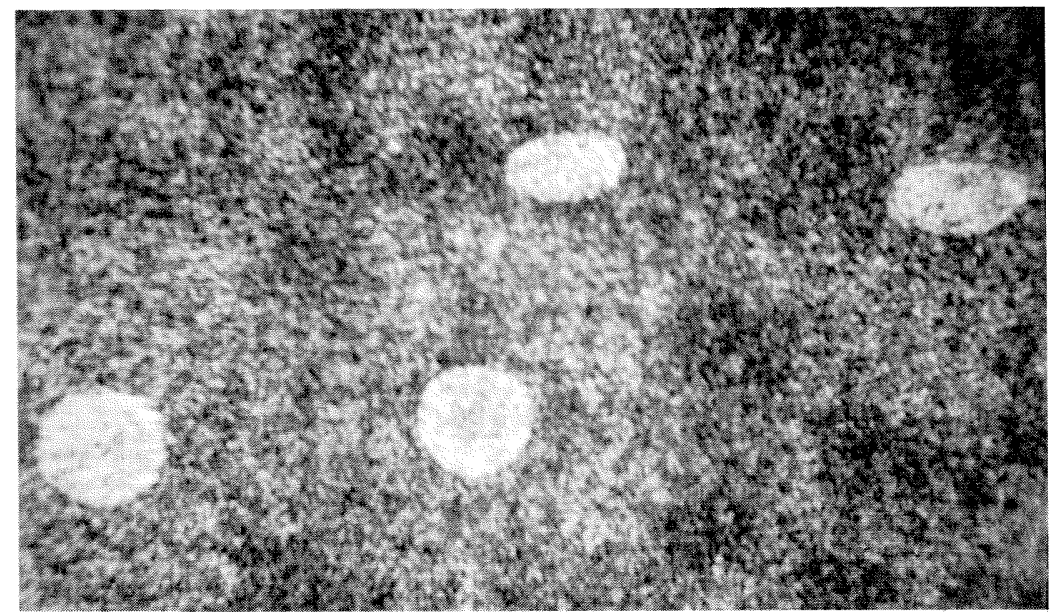

(c)

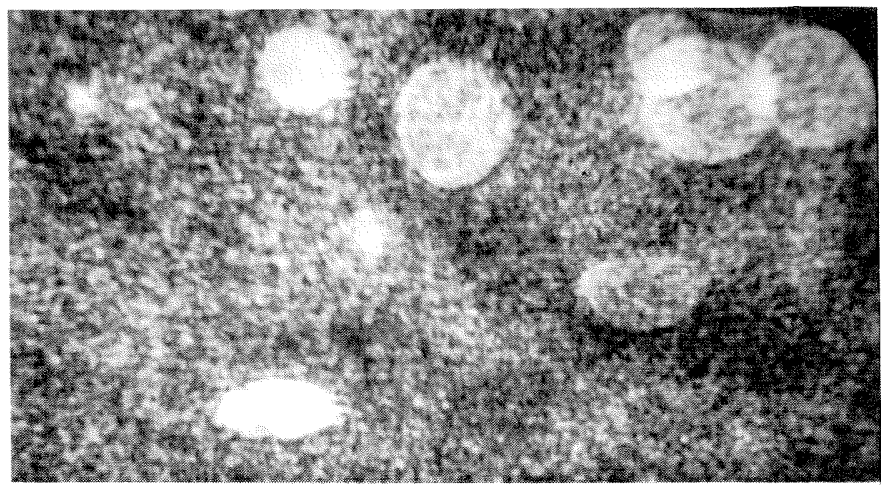

Figure 21. Double-pulsed hologram of bubble near core. (a) $x / c=2, \alpha=10^{\circ}, \operatorname{Re}=6.83 \times 10^{5}$. The vortex centreline is indicated by the multiple bubbles at the bottom of the photograph. The bubble at the top of the photograph is located at $R / c=0.011$. (b) $x . c=2, \alpha=5^{\circ}, R e=6.83 \times 10^{5}$. The vortex centreline is indicated by the spherical bubble in the bottom half of the photograph. The bubble at the top of the photograph is located at $R / c=0.004$. (c) $x / c=2, \alpha=5^{\circ}, R e=6.83 \times 10^{5}$. The vortex centreline is indicated by the bubbles at the top of the photograph. The bubble at the bottom of the photograph is located at $R / c=0.003$. In $(a-c) U_{\infty}$ is left to right. Scale: $5 \mathrm{~cm} \equiv 1.86 \mathrm{~mm}$ in flow. 
(a)

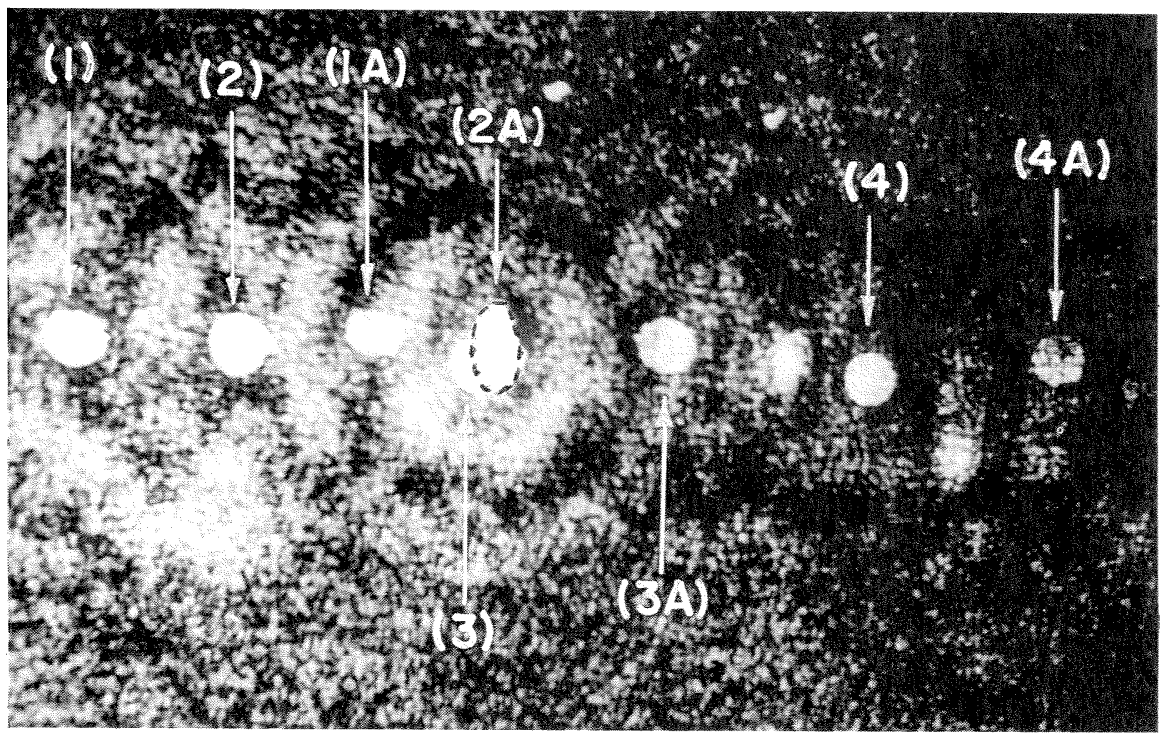

(b)

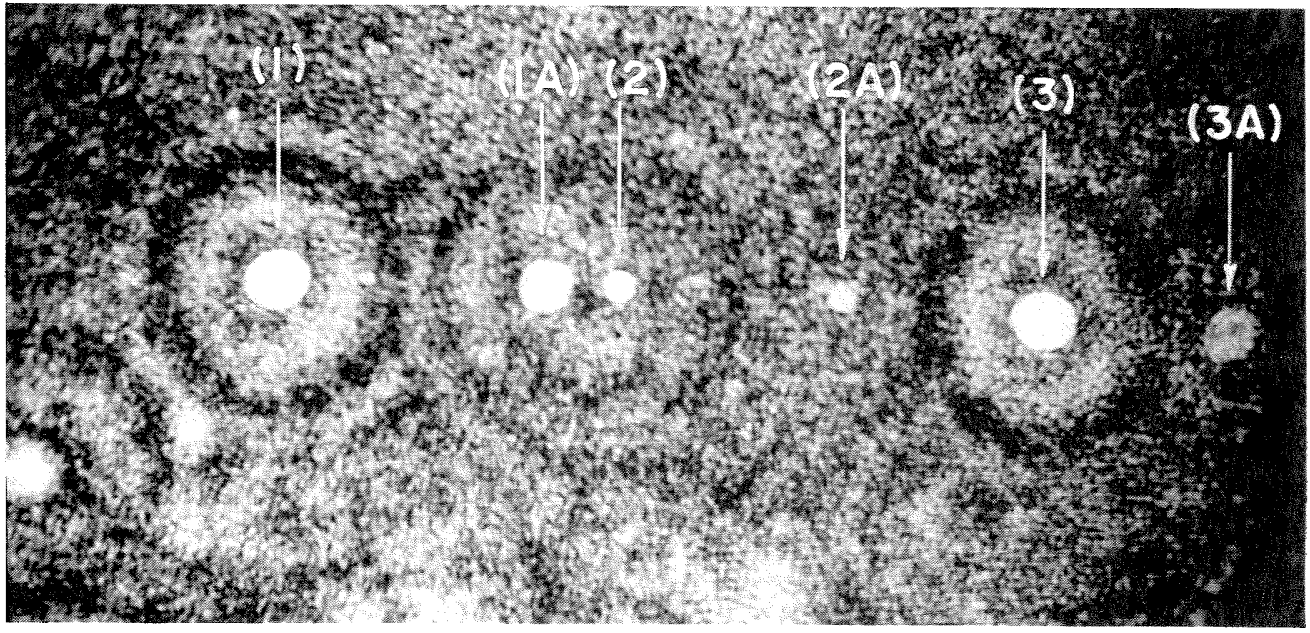

(c)

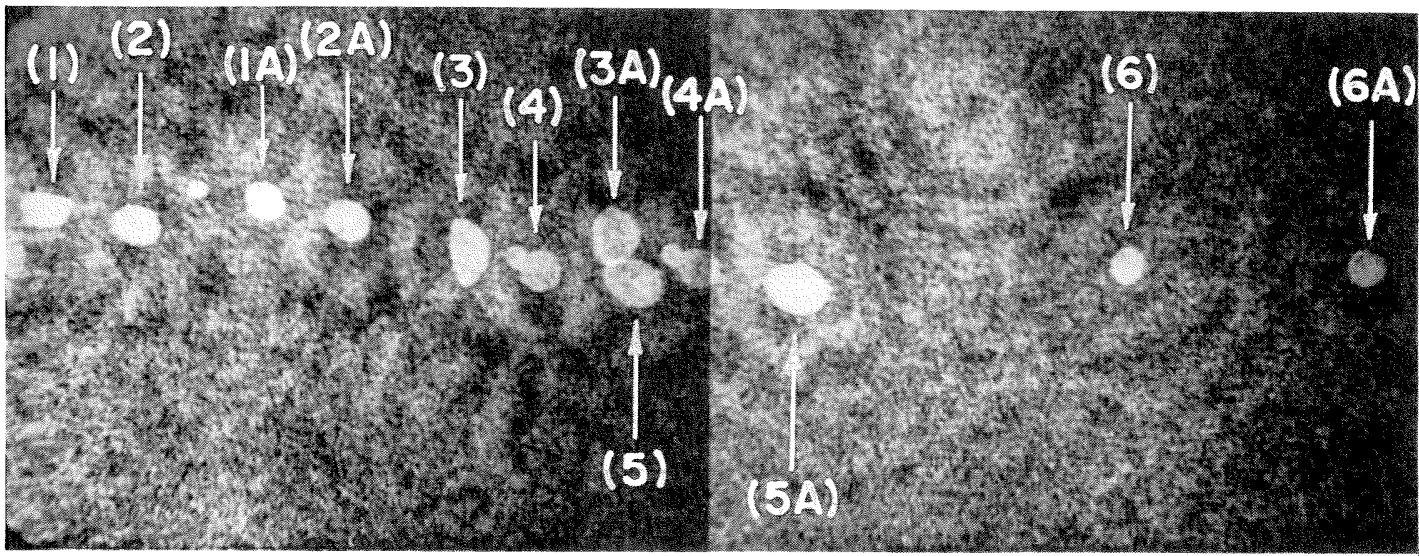

Fraure 22. For caption see facing page 
It is possible to establish a lower bound on the instantaneous axial accelerations in the trailing vortex. All such accelerations will be non-dimensionalized using the maximum centripetal acceleration around the core : $a_{\max }=\left[\left(U_{\theta}\right)_{\max }\right]^{2} / R_{\max }$. For the flow pictured in figure $22(c) a_{\max } \approx 7 \times 10^{3} \mathrm{~m} / \mathrm{s}^{2}$. Figure $22(a)$ is a typical doublepulsed hologram from which accelerations may be inferred. The bubble pair labelled 1 and $1 \mathrm{~A}$, to the left, represents a bubble moving at $1.65 U_{\infty}$. Bubble 2 has a speed of $1.43 U_{\infty}$, and bubbles 3 and 4 both are travelling at $1.05 U_{\infty}$. Bubble image $2 \mathrm{~A}$ shows the bubble to be highly deformed to an eccentricity of about 2 - suggestive of rapid deceleration of the core. If the region of rapid deceleration is assumed to be fixed in the Eulerian sense, and the bubble velocity is assumed to accurately reflect the local liquid velocity, then these velocities imply an axial deceleration of $0.60 U_{\infty}$ in $200 \mu \mathrm{s}$ or $1.7 a_{\max }\left(1.5 \times 10^{4} \mathrm{~m} / \mathrm{s}^{2}\right)$. Figure $22(b)$ is another example of rapid core deceleration. With the aforementioned assumptions, the bubble velocities imply an axial velocity deceleration in the core of $1.0 a_{\max }\left(9.5 \times 10^{3} \mathrm{~m} / \mathrm{s}^{2}\right)$. Figure $22(c)$ is a final example of rapid deceleration in the core. For technical reasons two adjacent photographs of the same reconstructed hologram of the core were spliced together to yield this figure. The bubble velocities imply a flow deceleration of approximately $1.6 a_{\max }$ $\left(1.3 \times 10^{4} \mathrm{~m} / \mathrm{s}^{2}\right)$ in the region between bubbles 2 and 3 . Note the elongated first image of bubble 3 . The flow reaccelerates by $1.1 \alpha_{\max }\left(7.9 \times 10^{3} \mathrm{~m} / \mathrm{s}^{2}\right)$ between bubbles 5 and 6 .

The assumption that the bubble velocity is equal to the local liquid velocity has been tested by using summary calculations of bubble motions in the core. The calculations consist of a quasi-static simulation of bubble motion which incorporates the force due to pressure gradients in the surrounding fluid, bubble drag, and added mass effects. These simulations suggest that the bubble velocity differs from that of the surrounding fluid by at most $20 \%$ for all flows studied, $\uparrow$ whence the axial deceleration must be in excess of the maximum centripetal acceleration around the centreline in some cases.

A third vortex feature which has been observed many times for large Reynolds numbers $\left(>7 \times 10^{5}\right)$ at small values of $x / c$, is a feature which may be termed a 'vortex kink' (Green 1988a). Figure $23(a)$ is such a vortex kink. The vortex centreline fluid travels in a straight line (indicated by the collinear first and second bubble images to the left) in the free-stream flow direction until the start of the kink (approximately $\frac{2}{5}$ of the distance from the left edge of the photograph). It then undergoes a rapid turning and cross-stream acceleration until the centreline is aligned at approximately $25^{\circ}$ with the free-stream flow, as evidenced by the central elongated bubble images. The centreline fluid at the end of the kink rapidly decelerates in the cross-stream direction and resumes motion parallel to the freestream direction. The kink is seen to propagate along the core with a velocity different from the free-stream velocity. Figure $23(b)$ is a second example of a vortex kink. Elongated bubbles in the centre of the photograph again reveal a cross-stream

$\dagger$ It should be noted that $20 \%$ is the maximum axial velocity error. $95 \%$ or more of the individual velocity measurements on the core were taken in regions of such small axial pressure gradient that the velocity difference between the bubbles and the surrounding water was negligible.

Figure 22. Double-pulsed hologram of bubbles on the vortex centreline. $(a) x / c=2, \alpha=10^{\circ}$, $R e=7.86 \times 10^{5}$. The two images of each bubble are labelled with a number and the number with an $\mathrm{A}$. The profile of bubble $2 \mathrm{~A}$ is dashed in, and is clearly visible in the reconstructed hologram image. Scale : $5 \mathrm{~cm} \equiv 1.86 \mathrm{~mm}$ in flow. $(b) x / c=2, \alpha=10^{\circ}, R e=7.86 \times 10^{5} .5 \mathrm{~cm} \equiv 2.44 \mathrm{~mm}$ in flow . (c) $x / c=2, \alpha=10^{\circ}, R e=6.83 \times 10^{5} .5 \mathrm{~cm} \equiv 2.79 \mathrm{~mm}$ in flow. $U_{\infty}$ is left to right. 
(a)

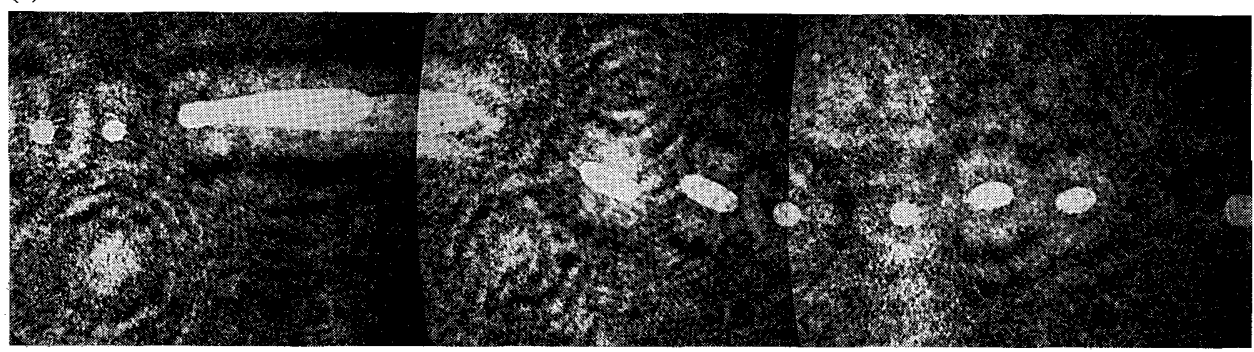

(b)

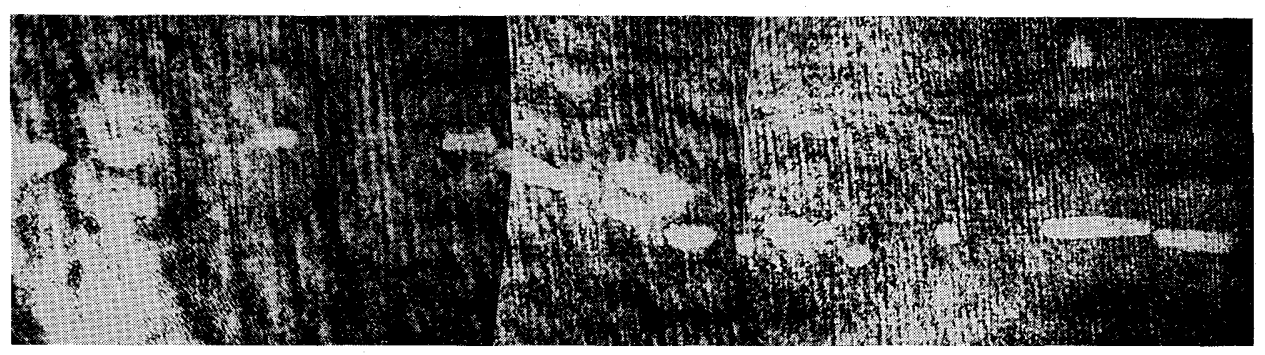

(c)

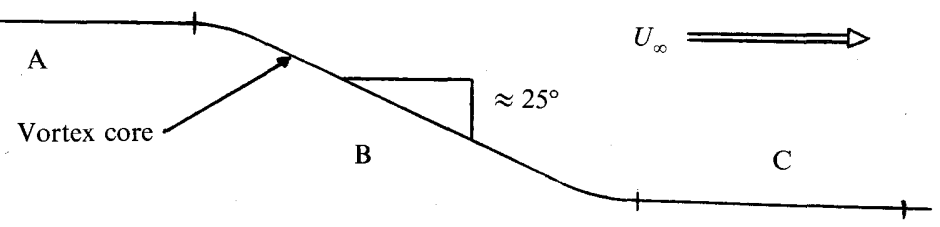

Figure 23 $(\alpha)$ Double-pulsed hologram of a vortex kink. $x / c=3, \alpha=5^{\circ}, R e=10^{6}$. $U_{\infty}$ is left to right. Scale: $5 \mathrm{~cm} \equiv 2.84 \mathrm{~mm}$ in flow. $(b)$ Double-pulsed hologram of a vortex kink. $x / c=2$, $\alpha=10^{\circ}, R e=1.03 \times 10^{5} . U_{\infty}$ is left to right. $5 \mathrm{~cm} \equiv 2.40 \mathrm{~mm}$ in flow. (c) Schematic of a vortex kink. $U_{x \mathrm{c}}$ may be as large as $2\left(\overline{U_{x \mathrm{e}}^{\prime}}\right)$ greater than the mean core velocity in either or both of regions $\mathrm{A}$ and $\mathrm{C}$. The axial velocity in region $\mathrm{B}$ is less than that in region $\mathrm{A}$.

angle in the kink of approximately $25^{\circ}$. The holographic reconstruction process is not sufficiently accurate to measure a swirl component of the kink, although kinks out of the holographic plane have been observed. Figure $23(c)$ is a schematic drawing of a typical vortex kink, based on these and similar photographs.

Vortex kinking is such an interesting phenomenon that the authors were intrigued to know if other researchers have noticed similar vortex behaviour. Hopfinger, Browand \& Gagne (1982) describes the flow in a deep, rotating tank fitted with a bottom-mounted turbulence generation grid. Concentrated vortices with axes roughly parallel to the rotation axis are an important feature of this flow. These concentrated vortices 'support waves consisting of helical distortions, which travel along the axes of individual vortices. Isolated, travelling waves seem well described by the vortex-solition theory of Hasimoto (1972)' (Hopfinger et al. 1982). The parallelism between this description and the vortex kinking observed in the present study is striking. This similarity of phenomena between two ostensibly very different vortical flows raises the possibility that Hasimoto type vortex kinks occur in many vortical flows. Vortex kinking does not seem to correspond with any of the types of vortex breakdown described by Leibovich (1978); flow reversal, which is a prerequisite for vortex breakdown, has never been observed. 


\section{Summary}

The tip vortex generated by a small-aspect-ratio rectangular planform hydrofoil in a water tunnel has been studied. Microbubbles were injected upstream of the wing. Some, having been driven by a centripetal pressure gradient force onto the vortex centreline, accurately marked its location; other microbubbles travelled around the core and served as nearly Lagrangian flow tracers. Double-pulsed holograms yielded tangential and axial velocity distributions around the vortex centreline. The bubble locations also provided some information about the global flow structure of the core.

The axial velocity in the vortex is highly unsteady; the unsteadiness increases rapidly as the centreline is approached. Centreline fluctuating r.m.s. axial velocities as large as $0.20 U_{\infty}$ have been measured. These fluctuating velocities are normally distributed. Very long-wavelength ('slow') axial velocity unsteadiness has been observed at $\alpha=10^{\circ}$ but, surprisingly, not at $\alpha=5^{\circ}$. The normalized mean axial velocity distribution, $\overline{U_{x}(R)} / U_{\infty}$, is 1 for $R / c>0.04$ and rises or falls rapidly inside this radius to the centreline value. The mean centreline axial velocity rises as $\alpha$ increases and decreases with increasing downstream distance. Furthermore, the centreline axial velocity is nearly Reynolds-number independent at small downstream distances, but is a strong function of the Reynolds number at larger values of $x / c$.

The normalized mean tangential velocity distribution, $\overline{U_{\theta}(R)} / U_{\infty}$, is nearly independent of both Reynolds number and downstream distance $\left(2 \times 10^{5} \leqslant R e \leqslant 10^{6}\right.$ and $2 \leqslant x / c \leqslant 10$ ), which means vortex rollup occurs over a very short distance and vorticity diffusion is extremely slow. $U_{\theta}$ achieves a maximum of $0.93 U_{\infty}$ (for $\alpha=10^{\circ}$ ) near $R / c=0.02$. The tangential velocity is $\theta$-independent; the vortex is axisymmetric to within the experimental error. The unsteady component of the tangential velocity falls significantly with downstream distance.

Trailing vortex core unsteadiness often takes the form of regions of rapid deceleration and acceleration separated by long expanses of relatively uniform velocity. Very large axial decelerations, larger even than the maximum centripetal acceleration around the core, have been observed. Flows at high Re with large core velocity excesses have vortex kinks similar to the vortex kinks observed by Hopfinger et al. (1982).

The Office of Naval Research has provided support under contract number N000167-85-K-0165. This research was carried out while the lead author was a graduate student, and subsequently a postdoctoral fellow, at Caltech. The authors appreciate the helpful comments of the reviewers.

\section{REFERENCES}

Baker, G. R., Barker, S. J., Bofah, K. K. \& Saffman, P. G. 1974 Laser anemometer measurements of trailing vortices. J. Fluid Mech. 65, 325-336.

BatcheloR, G. K. 1964 Axial flow in trailing line vortices. J. Fluid Mech. 20, 645-658.

Batchelor, G. K. 1967 An Introduction to Fluid Dynamics. Cambridge University Press.

Chigrer, N. A. 1974 Vortexes in aircraft wakes. Sci. Am. 245, 76-83.

Chigrer, N. A. \& Corsiglia, V. R. 1971 Tip vortices - velocity distributions. NASA TMX-62087.

Chigier, N. A. \& Corsiglia, V. R. 1972 Wind tunnel studies of wing wake turbulence. J. Aircraft 9, 820-825.

Corsiglia, V. R., Schwind, R. G. \& Chigier, N. A. 1973 Rapid scanning, three-dimensional hotwire anemometer surveys of wing-tip vortices. J. Aircraft 10, 752-757. 
Crow, S. C. 1970 Stability theory for a pair of trailing vortices. AIAA J. 8, 2172-2179.

Durand, W. F. (ed.) 1963 Aerodynamic Theory, vol. III, pp. 280-306. Dover.

GATES, E. M. 1977 The influence of free-stream turbulence, free-stream nuclei populations and a drag-reducing polymer on cavitation inception on two axisymmetric bodies. Ph.D. Thesis, California Institute of Technology.

Govindaraju, S. P. \& Saffman, P. G. 1971 Flow in a turbulent trailing vortex. Phys. Fluids 14, 2074-2080.

GREen, S. I. 1988 a Trailing vortex core unsteadiness - an exploratory study of Reynolds number effects. AIAA/ASME National Fluid Dynamics Congress, pp. 1064-1068.

Green, S. I. $1988 b$ Tip vortices - single phase and cavitating flow phenomena. Ph.D. thesis, California Institute of Technology.

Green, S. I. 1989 Tailored air bubble determination of trailing vortex core pressure. ASME Cavitation and Multiphase Flow Forum, San Diego.

Hasimoto, H. 1972 A soliton on a vortex filament. J. Fluid Mech. 51, 477-485.

Higuchi, H., Quadrelli, J.C. \& FarReli, C. 1987 Vortex roll-up from an elliptical wing at moderately low Reynolds numbers. AIAA J. 25, 1537-1542.

Hoffman, E. R. \& Joubert, P. N. 1963 Turbulent line vortices. J. Fluid Mech. 16, 395-411.

Holman, J. P. \& Moore, G. D. 1961 An experimental study of vortex chamber flow. Trans. ASME D: J. Basic Engng 83, 632.

Hopfinger, E. J., Browand, F. K. \& GaGne, Y. 1982 Turbulence and waves in a rotating tank. J. Fluid Mech. 125, 505-534.

JARvinen, P. O. 1973 Aircraft wing tip modification. J. Aircraft 10, 63-64.

Kreyszig, E. 1972 Advanced Engineering Mathematics. John Wiley and Sons.

Leibovich, S. 1978 The structure of vortex breakdown. Ann. Rev. Fluid Mech. 10, 221-246.

Logan, A. H. 1971 Vortex velocity distributions at large downstream distances. J. Aircraft 8, 930-932.

Mason, W. H. \& Marchman, J. F. 1972 The farfield structure of aircraft wake turbulence. AIAA Paper 72-40.

Moore, D. W. \& Saffman, P. G. 1973 Axial flow in laminar trailing vortices. Proc. $R$. Soc. Lond. A 333, 491-508.

ORLOFF, K. L. 1971 Experimental investigation of upstream influence in a rotating flowfield. Ph.D. thesis, University of California, Santa Barbara.

Orloff, K. L. \& Grant, G. R. 1973 The application of a scanning laser doppler velocimeter to trailing vortex definition and alleviation. AIAA Paper 73-680.

Phildips, W. R. C. 1981 The turbulent trailing vortex during roll-up. J. Fluid Mech. 105, 451-467.

Saffman, P. G. 1973 Structure of turbulent line vortices. Phys. Fluids 16, 1181-1188.

Singh, P. I. \& Uberoi, M. S. 1976 Experiments on vortex stability. Phys. Fluids 19, 1858-1863.

Spreiter, J. R. \& Sacks, A. H. 1951 The rolling up of the trailing vortex sheet and its effect on the downwash behind wings. J. Aero. Sci. 18, 21-32.

Stinebring, D. R., Farrell, K. J. \& Billet, M. L. 1989 Structure of a tip vortex trailing from a three-dimensional hydrofoil. 22nd American Towing Tank Conf., St. John's, Canada.

Tномpson, D. H. 1975 Experimental study of axial flow in wing tip vortices. J. Aircraft 12, 910-911.

Thwaites, B. (ed.) 1960 Incompressible Aerodynamics pp. 325-347. Dover.

ZALAY, A. D. 1976 Hot-wire and vorticity meter wake vortex surveys. AIAA J. 15, 694-696. 